\title{
Cálculo Funcional Holomorfo para Operadores Pseudodiferenciais
}

\author{
Marco Eduardo Barros Chucata
}

DISSERTAÇÃO APRESENTADA

$\mathrm{AO}$

Instituto De Matemática e Estatística

DA

UNIVERSIDADE DE SÃO PAUlO

PARA

OBTENÇÃO DO TÍTULO

$\mathrm{DE}$

Mestre EM CiênCIAS

Programa: Matemática Aplicada

Orientador: Prof. Dr. Pedro Tavares Paes Lopes

São Paulo, Julho de 2019 


\section{Cálculo Funcional Holomorfo para Operadores Pseudodiferenciais}

Esta versão da dissertação contém as correções e alterações sugeridas pela Comissão Julgadora durante a defesa da versão original do trabalho, realizada em 13/06/2019. Uma cópia da versão original está disponível no

Instituto de Matemática e Estatística da Universidade de São Paulo.

Comissão Julgadora:

- Prof. Dr. Pedro Tavares Paes Lopes (orientador) - IME/USP

- Prof. Dr. Rafael Augusto dos Santos Kapp - UFSCAR

- Prof. Dr. Tiago Henrique Picon - FFLRP/USP 


\section{Agradecimentos}

Agradeço ao meu orientador Prof. Dr. Pedro Tavares Paes Lopes pela orientação e pelo incentivo durante a realização deste trabalho. 


\section{Resumo}

CHUCATA, M. E. B. Cálculo Funcional Holomorfo para Operadores Pseudodiferenciais. 2019. Dissertação (Mestrado) - Instituto de Matemática e Estatística, Universidade de São Paulo, São Paulo, 2019.

O cálculo funcional de operadores em espaços de Banach tem uma longa história, sendo inicialmente desenvolvido por F. Riesz, N. Dunford entre outros. Em 1986, uma importante contribuição foi feita por Alan McIntosh, que definiu um cálculo funcional holomorfo de operadores setoriais e destacou uma importante classe desses operadores: a dos operadores com cálculo funcional holomorfo limitado (CFHL).

Do ponto de vista de operadores diferenciais e pseudodiferenciais, alguns elementos envolvidos neste cálculo já estavam presentes nos trabalhos de R. T. Seeley sobre potências complexas de operadores diferenciais elípticos. Mais tarde mostrou-se que diversos operadores possuem CFHL. Um artigo recente nesta direção e base para esta dissertação foi publicado por Bilyj, Schrohe e Seiler.

Neste trabalho mostraremos que certos operadores pseudodiferenciais, agindo em espaços de Banach apropriados, são setoriais e possuem CFHL. Para isso faremos o estudo da álgebra dos símbolos de ordem zero e utilizaremos uma construção para a parametriz do resolvente.

A apresentação procura ser uma versão mais didática do artigo de Bilyj, Schrohe e Seiler. Além disso, fazemos certas adaptações nas demonstrações com o propósito de facilitar a compreensão dos argumentos. Também vamos apresentar aplicações do resultado obtido.

Palavras-chave: Operador Pseudodiferencial, Cálculo Funcional, Operador Setorial. 


\section{Abstract}

\section{CHUCATA, M. E. B. Holomorphic Functional Calculus for Pseudodifferential Operators.}

2019. Dissertação (Mestrado) - Instituto de Matemática e Estatística, Universidade de São Paulo, São Paulo, 2019.

Functional calculus for operators acting on Banach Spaces has a long history. It was initially developed by F. Riesz, N. Dunford among others. In 1986, an important contribution was made by Alan McIntosh who defined a holomorphic functional calculus for sectorial operators and put on the scene an important class of sectorial operators, namely, operators with a bounded holomorphic functional calculus (BHFC).

From the point of view of differential and pseudodifferential operators, some elements treated in this calculus were already in the works of R. T. Seeley about complex powers of elliptic differential operators. Later it was shown that several operators have BHFC. A recent paper in this direction, and the one on which this dissertation is based, was published by Bilyj, Schrohe and Seiler.

In this work we show that certain pseudodifferential operators, acting on appropriate Banach spaces, are sectorial and have BHFC. For this we will study the algebra of symbols of order zero and use a construction for the parametrix.

This presentation aims to explore and detail the paper of Bilyj, Schrohe and Seiler. Furthermore, we make adaptations in the proofs in order to clarify the argument. We also show applications of the obtained results.

Keywords: Pseudodifferential Operator, Functional Calculus, Sectorial Operator. 


\section{Sumário}

$\begin{array}{ll}\text { Lista de Símbolos } & \text { ix }\end{array}$

1 Introdução $\quad 1$

2 Cálculo Funcional Holomorfo $\quad 3$

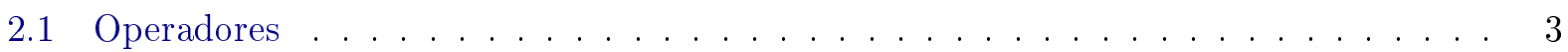

2.2 Integral de Bochner . . . . . . . . . . . . . . . . . . . 4

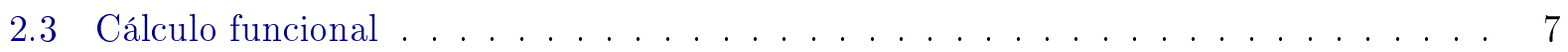

3 Operadores Pseudodiferenciais $\quad 13$

3.1 Preliminares . . . . . . . . . . . . . . . . . . . . . . . . 13

3.2 Operadores Pseudodiferenciais . . . . . . . . . . . . . . . . . . . . . 14

3.3 Álgebra dos símbolos . . . . . . . . . . . . . . . . . . . . . . . . . . . . . . . . . . . . . . . .

3.4 Inversa e $\psi$-Álgebra . . . . . . . . . . . . . . . . . . . . . . . . 18

4 Cálculo $H^{\infty}$ para operadores pseudodiferenciais $\quad 21$

4.1 Cálculo para o símbolo . . . . . . . . . . . . . . . . . . . 21

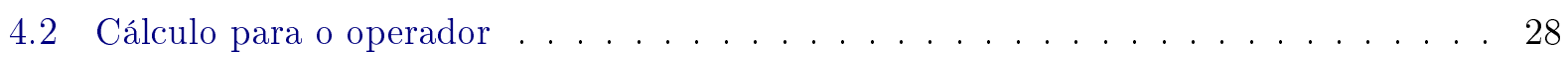

5 Aplicações $\quad 31$

5.1 Regularidade Maximal . . . . . . . . . . . . . . . . . . . . . . . 31

5.2 Aplicações . . . . . . . . . . . . . . . . . . . . . . . 32

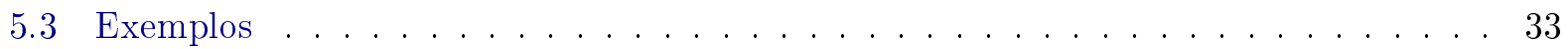

Referências Bibliográficas $\quad 35$ 
viii SUMÁRIO 


\section{Lista de Símbolos}

$\mathbb{N}_{0} \quad$ Conjunto dos inteiros $0,1,2, \ldots$

$\mathbb{N} \quad$ Conjunto dos inteiros positivos $1,2,3, \ldots$

$\mathcal{B}(X) \quad$ Espaço dos operadores lineares $A: X \rightarrow X$ contínuos num espaço de Banach $X$

$X^{*} \quad$ Espaço dos funcionais lineares $\varphi: X \rightarrow \mathbb{C}$ contínuos num espaço de Banach $X$

$\mathcal{S} \quad$ Espaço das funções de decaimento rápido em $\mathbb{R}^{n}$ (Espaço de Schwartz)

$H(\Omega, X) \quad$ Espaço das funções holomorfas $f: \Omega \subset \mathbb{C} \rightarrow X$ onde $X$ é um espaço de Banach $D_{j}=(-i) \partial_{j}$

$\Sigma_{\theta} \quad$ Conjunto $\left\{r e^{i \varphi} \in \mathbb{C}: r>0,-\theta<\varphi<\theta\right\}$ (setor aberto de ângulo $\theta$ ) 


\section{Capítulo 1}

\section{Introdução}

Nosso objetivo principal será estudar o cálculo funcional de operadores pseudodiferenciais hipoelípticos. Em particular, mostraremos que certos operadores pseudodiferenciais, agindo em espaços de Banach sujeitos a condições de hipoelipticidade, são setoriais e possuem cálculo $H^{\infty}$ limitado. Os resultados se baseiam em [BSS10], no entanto a apresentação procura ser mais didática. Além disso, fazemos certas adaptações nas demonstrações com o propósito de facilitar a compreensão dos argumentos.

Sob certo ponto de vista, a essência do resultado que apresentamos é mostrar que um operador concreto possui uma propriedade abstrata que, por sua vez, nos fornece mais ferramentas para estudar e resolver problemas envolvendo o operador concreto.

Para o nosso caso os operadores concretos são os operadores pseudodiferencias que generalizam os operadores diferenciais lineares. Os primeiros trabalhos que introduziram as técnicas e abordagem da teoria de operadores pseudodiferencias apareceram nas publicações de [H6̈5] e [KN65]. Uma das ideias mais importantes da teoria consiste em estudar os operadores através da manipulação de seus respectivos símbolos.

O lado abstrato é formado pelo cálculo funcional. Dado um operador linear $A$, a ideia é associar para cada função $f$ um operador $f(A)$. Quando $A$ é um operador limitado definido num espaço de Banach, a Fórmula Integral de Cauchy fornece uma representação interessante e já permite provar teoremas espectrais importantes [Rud91]. Na prática, como o espectro de um operador limitado é compacto, podemos escolher uma curva $\Gamma$ contornando o espectro de $A$ e, para cada função holomorfa $f$, definir

$$
f(A)=\frac{1}{2 \pi i} \int_{\Gamma} f(\lambda)(\lambda-A)^{-1} d \lambda
$$

através da integral de Bochner. Uma referência clássica para o cálculo funcional de operadores limitados é o livro [DS58].

Nas aplicações muitas vezes lidamos com operadores lineares não limitados cujo espectro, em geral, é um conjunto ilimitado de $\mathbb{C}$. Veremos que no caso de operadores setoriais (com a mesma definição usada em [Yag10]) ainda conseguimos aproveitar a integral acima para definir $f(A)$ (como operador limitado) desde que façamos uma restrição sobre a classe de funções à qual $f$ pertence. Quando esta associação $f \mapsto f(A)$ possui uma extensão linear ao espaço de funções holomorfas limitadas num setor e satisfaz $f g(A)=f(A) g(A)$ e

$$
\|f(A)\| \leq C\|f\|_{\infty},
$$

diremos que $A$ possui cálculo $H^{\infty}$. O trabalho que fundou as bases do cálculo funcional para operadores setoriais encontra-se em [McI86].

Selecionando funções específicas em $H^{\infty}$ recuperamos operadores importantes no estudo de problemas envolvendo $A$. Por exemplo, tomando $f_{t}(z)=z^{i t}$ obtemos que as potências imaginárias de $A$ são limitadas e satisfazem a estimativa $\left\|A^{i t}\right\| \leq C e^{\theta t}$ para certo $C>0$ e $\theta$ independentes de $t$. Por sua vez o teorema de Dore e Venni [DV87] implica existência e unicidade de equações de 
evolução envolvendo $A$, se $\theta<\pi / 2$.

Os operadores pseudodiferencias que abordamos possuem símbolos nas classes $S_{\rho, \delta}^{m}$ introduzidas em [H6̈7]. Especificamente os símbolos desta classe satisfazem as estimativas globais

$$
\left|D_{\xi}^{\alpha} D_{x}^{\beta} a(x, \xi)\right| \leq C_{\alpha, \beta}\langle\xi\rangle^{m-\rho|\alpha|+\delta|\beta|}
$$

em $\mathbb{R}_{\xi}^{n} \times \mathbb{R}_{x}^{n}$. O operador correspondente age no espaço de Schwartz $\mathcal{S}$ e tem a forma

$$
o p[a] u(x)=(2 \pi)^{-n} \int_{\mathbb{R}^{n}} e^{i x \cdot \xi} a(x, \xi) \hat{u}(\xi) d \xi
$$

onde $\hat{u}$ é a transformada de Fourier de $u$.

Normalmente fixamos um espaço de funções $E$ que possui uma estrutura de espaço de Banach e contém $\mathcal{S}$ como subconjunto denso $\left(E=L^{p}\right.$, por exemplo). Deste modo, cada operador op $[a]$ pode ser visto como um operador linear não limitado em $E$. Colocando certas hipóteses sobre o símbolo $a$ será provado no Capítulo 4 que o fecho de op $[a]$ é um operador setorial com cálculo $H^{\infty}$.

A dissertação é organizada da seguinte maneira. No Capítulo 2 relembramos alguns fatos sobre espaços de Banach e operadores lineares não limitados. Em seguida, damos definições e resultados relevantes sobre a integral de Bochner com a intenção de transferir teoremas clássicos sobre funções escalares para funções vetoriais (por exemplo, o Teorema de Cauchy). Finalizamos o capítulo com uma introdução ao cálculo funcional de operadores setoriais e com a demonstração do Teorema 2.23 que fornece uma condição necessária e suficiente para um operador setorial ter cálculo $H^{\infty}$.

No Capítulo 3 é feito um apanhado de resultados da teoria de operadores pseudodiferenciais: definição dos símbolos e operadores, expansão assintótica, composição, adjunto, etc. Com esta base, mostramos que a classe de símbolos de ordem zero, $S_{\rho, \delta}^{0}(0 \leq \delta<\rho \leq 1)$, é uma álgebra de Fréchet tomando como multiplicação o símbolo da composição. Enunciamos um teorema sobre a álgebra $S_{\rho, \delta}^{0}$ que permite provar dois resultados importantes para nossa demonstração do teorema principal: o grupo de invertíveis da álgebra $S_{\rho, \delta}^{0}$ é aberto e a inversão é contínua.

No Capítulo 4 provamos detalhadamente o principal resultado no Teorema 4.12 que dá condições suficientes para um operador pseudodiferencial ter um cálculo $H^{\infty}$ limitado.

Os resultados apresentados na dissertação, apesar de sua aparência bastante abstrata, possuem diversas aplicações, em especial na teoria de equações parabólicas não lineares. Além do Teorema de Dore e Venni, o cálculo funcional possui importantes consequências para a teoria de interpolação complexa. Diversas aplicações do cálculo funcional e da regularidade maximal podem ser encontradas, por exemplo, no livros de Haase [Haa06] e Prüss e Simonett [PS16]. 


\section{Capítulo 2}

\section{Cálculo Funcional Holomorfo}

Vamos apresentar neste capítulo os conceitos básicos sobre medida, integração e análise funcional necessários para a dissertação e para a compreensão do cálculo funcional.

Na Seção 2.1 recordamos algumas definições básicas sobre operadores lineares e fixamos certas notações.

Na Seção 2.2 fazemos uma introdução sucinta à integral de Bochner para funções tomando valores em espaços de Banach. Discutimos aqui apenas os resultados necessários para desenvolver o cálculo funcional. Como consequência obtemos os teoremas da análise complexa no caso de funções holomorfas vetorias e, além disso, provamos versões destes resultados para uma classe de curvas não limitadas.

Concluímos, na seção 2.3, com uma introdução ao cálculo funcional de operadores setorias. Nesta parte definimos o cálculo $H^{\infty}$ e damos uma condição necessária e suficiente para sua existência.

\subsection{Operadores}

Seja $X$ um espaço vetorial sobre $\mathbb{C}$.

Definição 2.1. Uma seminorma em $X$ é uma função $p: X \rightarrow \mathbb{R}$ satisfazendo

- $p \geq 0$

- $p(\alpha x)=|\alpha| p(x)$

- $p(x+y) \leq p(x)+p(y)$

Se, além disso, tivermos

- $p(x)=0 \Rightarrow x=0$

dizemos que p é uma norma.

Usualmente escrevemos $\|x\|_{X}$ ou $\|x\|$ no lugar de $p(x)$ quando $p$ é uma norma.

Definição 2.2. Um espaço de Banach é um espaço vetorial com uma norma tal que, com a métrica $d(x, y)=\|x-y\|$ o espaço $(X, d)$ é completo.

Um espaço de Fréchet será para nós um espaço localmente convexo, Hausdorff, metrizável e completo. No entanto, aqui é mais conveniente definir esta mesma estrutura através de seminormas.

Definição 2.3. Um espaço de Fréchet é um espaço vetorial F equipado com uma família enumerável de seminormas $\left\{p_{j}: j \in \mathbb{N}_{0}\right\}$ satisfazendo

- Para cada $0 \neq x \in F$ existe $j \in \mathbb{N}_{0}$ tal que $p_{j}(x) \neq 0$. 
- Com a métrica

$$
d(x, y)=\sum_{j=0}^{\infty} 2^{-j} \frac{p_{j}(x-y)}{1+p_{j}(x-y)}
$$

o espaço $(F, d)$ é completo.

Observamos que todo espaço de Banach é de Fréchet.

Finalizamos esta seção definindo e enunciando resultados ligados aos operadores lineares.

Definição 2.4. Seja $X$ um espaço de Banach. Uma função $A: D(A) \rightarrow X$ é um operador (linear) se $D(A)$ é subespaço de $X$ e $A$ é linear.

- $O$ operador $A$ é fechado se o gráfico $G(A)=\{(x, y) \in X \times X: x \in D(A), A x=y\}$ é um subconjunto fechado de $X \times X$.

- $O$ operador $A$ é densamente definido se $\overline{D(A)}=X$.

- $O$ operador $A$ é limitado se $D(A)=X$ e $\|A\| \doteq \sup \left\{\|A x\|_{X}: x \in D(A),\|x\|_{X}=1\right\}$ é finito. Neste caso escrevemos $A \in \mathcal{B}(X)$.

Definição 2.5. Seja $A$ um operador linear. O conjunto formado por $\lambda \in \mathbb{C}$ tais que $\lambda-A: D(A) \rightarrow$ $X$ é bijetor com inversa $(\lambda-A)^{-1} \in \mathcal{B}(X)$, é chamado resolvente de $A$ e é denotado $\rho(A)$. O espectro de $A$ é o conjunto $\sigma(A) \doteq \mathbb{C} \backslash \rho(A)$.

Proposição 2.6. Seja A um operador fechado. Então, para $\lambda$ e $\mu$ em $\rho(A)$, temos:

- $O$ conjunto $\rho(A)$ é aberto em $\mathbb{C}$ (portanto $\sigma(A)$ é fechado) e o disco aberto $B(\lambda, \|(\lambda-$ $\left.A)^{-1} \|^{-1}\right) \subset \rho(A)$;

- A aplicação $\lambda \in \rho(A) \mapsto(\lambda-A)^{-1}$ é contínua (e até analítica);

- $(\mu-\lambda)(\lambda-A)^{-1}(\mu-A)^{-1}=(\lambda-A)^{-1}-(\mu-A)^{-1}$ (identidade do resolvente);

- Os operadores $(\lambda-A)^{-1}$ e A comutam, isto é, $(\lambda-A)^{-1} A x=A(\lambda-A)^{-1} x$ para todo $x \in D(A)$;

- $A(\lambda-A)^{-1}=\lambda(\lambda-A)^{-1}-I \in \mathcal{B}(X)$.

\subsection{Integral de Bochner}

Nesta seção fixamos $I$ um intervalo de $\mathbb{R}$ e $X$ um espaço de Banach. Denotamos por $\mathcal{A}$ a coleção dos subconjuntos de $I$ Lebesgue mensuráveis e $\mu$ denota a medida de Lebesgue. Nosso propósito é definir a integral de funções $f: I \rightarrow X$ no sentido de Bochner. A referência principal para as demonstrações dos resultados sobre esta integral é o livro do Arendt [ABHN11] (Capítulo 1, Seção 1.1). As notas de aulas do D. V. Tausk [Tau05] e o livro do Yosida [Yos95] são boas referências para o assunto onde a teoria é desenvolvida em espaços de medida mais gerais.

Definição 2.7. Dizemos que uma função $f: I \rightarrow X$ é simples se $f(I)$ é finito e para todo $c \in$ $f(I) \backslash\{0\}$ temos $f^{-1}(c) \in \mathcal{A}$ e $\mu\left(f^{-1}(c)\right)<\infty$. Neste caso temos a identidade $f=\sum_{c \in f(I)} c \chi_{f^{-1}(c)}$ e definimos a integral como a seguinte soma

$$
\int_{I} f d \mu=\sum_{c \in f(I) \backslash\{0\}} c \mu\left(f^{-1}(c)\right)
$$

Diremos que uma sequência de funções $f_{n}: I \rightarrow X$ converge quase sempre para uma função $f: I \rightarrow X$ se existir um conjunto $N \in \mathcal{A}$ de medida nula tal que $\lim _{n \rightarrow \infty} f_{n}(x)=f(x)$ para todo $x \in I \backslash N$. 
Definição 2.8. Uma função $f: I \rightarrow X$ é fortemente mensurável se existir uma sequência de funçôes simples $\left(f_{n}\right)_{n \in \mathbb{N}}$ convergindo quase sempre para $f$.

Se $g: X \rightarrow \mathbb{C}$ é uma função contínua e $f: I \rightarrow X$ é fortemente mensurável então $g \circ f: I \rightarrow \mathbb{C}$ é Lebesgue mensurável. De fato, basta notar que $g \circ f$ é o limite das funções $g \circ f_{n}$ e que tais funções são Lebesgue mensuráveis. Em particular $\|f\|: I \rightarrow \mathbb{R}$ é Lebesgue mensurável.

Definição 2.9. Diremos que $f: I \rightarrow X$ é (Bochner) integrável se existir uma sequência de funções simples $\left(f_{n}\right)_{n \in \mathbb{N}}$ convergindo quase sempre para $f$ tais que $\lim _{n \rightarrow \infty} \int_{I}\left\|f_{n}-f\right\| d \mu=0$. Neste caso definimos $\int_{I} f d \mu=\lim _{n \rightarrow \infty} \int_{I} f_{n} d \mu$ e denotamos por $L^{1}(I ; X)$ o conjunto das funções integráveis módulo funções que se anulam quase sempre.

Quando $X$ é $\mathbb{R}$ ou $\mathbb{C}$ a definição acima equivale a integrabilidade no sentido de Lebesgue. Para decidir quando uma função é Bochner integrável, em geral, usamos o critério a seguir.

Proposição 2.10. Uma função $f: I \rightarrow X$ é Bochner integrável se, e somente se, $f$ é fortemente mensurável e $\|f\|: I \rightarrow \mathbb{R}$ é Lebesgue integrável.

As funções $f: I \rightarrow X$ de nosso interesse serão contínuas e, portanto, fortemente mensuráveis. Desse modo, a fim de mostrar que uma função contínua é integrável, basta checar se sua norma é integrável.

Enunciaremos a seguir as propriedades relevantes da integral de Bochner.

Proposição 2.11. O conjunto de funções $L^{1}(I ; X)$ é um espaço vetorial e a integral $s: L^{1}(I ; X) \rightarrow$ $X, s(f)=\int_{I} f d \mu$, é linear e satisfaz a desigualdade:

$$
\left\|\int_{I} f d \mu\right\| \leq \int_{I}\|f\| d \mu
$$

Teorema 2.12 (Convergência Dominada). Seja $f_{n}: I \rightarrow X$ uma sequência de funçôes integráveis convergindo quase sempre para uma função $f: I \rightarrow X$. Se existir $q: I \rightarrow[0,+\infty]$ integrável tal que $\left\|f_{n}\right\| \leq q$ para todo $n$ então $f$ é integrável $e$

$$
\int_{I} f d \mu=\lim _{n \rightarrow \infty} \int_{I} f_{n} d \mu
$$

Proposição 2.13. Sejam $A: D(A) \subset X \rightarrow Y$ um operador fechado e $f: I \rightarrow X$ integrável com $f(I) \subset D(A)$. Se Af:I $\rightarrow Y$ for integrável então $\int_{I} f d \mu \in D(A) e$

$$
A \int_{I} f d \mu=\int_{I} A f d \mu
$$

Apresentamos brevemente outros espaços de funções, definidas num intervalo $I=] 0, T[$, relevantes para o capítulo de aplicações.

O espaço $L^{p}(I ; X)$ é formado pelas funções $f: I \rightarrow X$ fortemente mensuráveis tais que $\|f\|_{p, X}=$ $\left(\int_{I}\|f(s)\|^{p} d s\right)^{1 / p}<\infty$. Como é usual fazemos a identificação de funções que coincidem quase sempre. Neste caso $\|f\|_{p, X}$ é uma norma e $\left(L^{p}(I ; X),\|\cdot\|_{p, X}\right)$ é um espaço de Banach.

Outro espaço importante é o espaço de Sobolev $W_{p}^{1}(I ; X)$ formado pelas funções $f \in L^{p}(I ; X)$ para as quais existe $g \in L^{p}(I ; X)$ satisfazendo

$$
\int_{I} g(s) u(s) d s=-\int_{I} f(s) u^{\prime}(s) d s
$$

para toda $u \in C_{c}^{\infty}(] 0, T[; \mathbb{C})$. A função $g$ é única módulo funções que se anulam quase sempre. Para ver isso suponha que $h$ satisfaz a equação integral acima. Logo $\int_{I}(g(s)-h(s)) u(s) d s=0$ para todo $u \in C_{c}^{\infty}(] 0, T[; \mathbb{C})$. Dado $\varphi \in X^{*}$ um funcional linear contínuo temos, pela Proposição 2.13, que $\int_{I} \varphi((g(s)-h(s))) u(s) d s=0$. Assim, $\varphi((g(s)-h(s)))=0$ quase sempre e, por Hahn-Banach, 
obtemos que $g(s)-h(s)=0$ quase sempre. Denotamos $f^{\prime}=g$. Em termos de distribuições estamos dizendo que $f, f^{\prime} \in L^{p}(I ; X)$. Aqui a norma $\|f\|_{W_{p}^{1}}=\|f\|_{p, X}+\left\|f^{\prime}\right\|_{p, X}$ torna $\left(W_{p}^{1}(I ; X),\|\cdot\|_{W_{p}^{1}}\right)$ um espaço de Banach.

Podemos mostrar que cada $f \in W_{p}^{1}(I ; X)$ possui um representante contínuo $\bar{f} \in C(I ; X)$ dado por

$$
\bar{f}(t)=\bar{f}(0)+\int_{0}^{t} f^{\prime}(s) d s
$$

Logo faz sentido falar na função $f: I \rightarrow X$ e de seus valores $f(t)$.

A seguir vamos apresentar versões vetoriais de alguns teoremas de análise complexa. Seja $X$ um espaço de Banach complexo e $\Omega \subset \mathbb{C}$ aberto. Uma função $f: \Omega \rightarrow X$ é dita holomorfa (em $\Omega$ ) se para todo $z_{0} \in \Omega$ existe o limite

$$
f^{\prime}\left(z_{0}\right) \doteq \lim _{z \rightarrow z_{0}} \frac{f(z)-f\left(z_{0}\right)}{z-z_{0}} \in X
$$

É fácil ver que toda função holomorfa é contínua e se $\varphi \in X^{*}$ e $f: \Omega \rightarrow X$ é holomorfa, então $\varphi \circ f: \Omega \rightarrow \mathbb{C}$ é holomorfa (escalar) com derivada igual a $(\varphi \circ f)^{\prime}(z)=\varphi\left(f^{\prime}(z)\right)$.

Lembramos que uma curva $C^{1}$ em $\Omega$ é uma função $\gamma:[a, b] \rightarrow \Omega$ de classe $C^{1}$ e dizemos curva fechada se $\gamma(a)=\gamma(b)$. O índice de uma curva $\gamma$ com relação a um ponto $\alpha \in \mathbb{C} \backslash \gamma([a, b])$ é definido pela integral

$$
\operatorname{ind}(\gamma, \alpha)=\frac{1}{2 \pi i} \int_{\gamma} \frac{d z}{z-\alpha}
$$

Uma colecão finita de curvas será chamada de cadeia e denotada por $\Gamma=\gamma_{1} \dot{+} \ldots \dot{+} \gamma_{k}$. Um ciclo é uma cadeia formada apenas por curvas fechadas. O índice de uma cadeia é a soma dos índices de suas componentes.

Se $f: \Omega \rightarrow X$ é contínua e $\gamma$ é uma curva $C^{1}$ em $\Omega$, definimos a integral de $f$ sobre $\gamma$ pela integral de Bochner

$$
\int_{\gamma} f=\int_{[a, b]} f(\gamma(t)) \gamma^{\prime}(t) d t
$$

que fica bem definida neste caso, pois $g(t)=f(\gamma(t)) \gamma^{\prime}(t), t \in[a, b]$ é contínua e sua norma $\|g(t)\|=$ $\|f(\gamma(t))\|\left|\gamma^{\prime}(t)\right|$ é integrável no compacto $[a, b]$.

Análogo ao caso escalar definimos a integral sobre uma cadeia $\Gamma=\gamma_{1} \dot{+} \ldots \dot{+} \gamma_{k}$ pela soma

$$
\int_{\Gamma} f=\sum_{j=1}^{k} \int_{\gamma_{j}} f
$$

Teorema 2.14. Sejam $\Omega$ um aberto de $\mathbb{C}, f: \Omega \rightarrow X$ uma função holomorfa.

Se $\Gamma$ é um ciclo em $\Omega$ tal que para todo $\alpha \in \mathbb{C} \backslash \Omega$ vale

$$
\operatorname{ind}(\Gamma, \alpha)=0
$$

então

$$
\int_{\Gamma} f=0
$$

$e$

$$
\operatorname{ind}(\Gamma, \alpha) f(\alpha)=\frac{1}{2 \pi i} \int_{\Gamma} \frac{f(z)}{z-\alpha} d z
$$

para $\alpha \in \Omega \backslash \Gamma$.

Demonstração. Seja $\varphi \in X^{*}$. Como consequência da Proposição 2.13 e pelo Teorema de Cauchy 
escalar aplicado a função $\varphi \circ f$ temos

$$
\varphi\left(\int_{\Gamma} f\right)=\int_{\Gamma} \varphi \circ f=0
$$

para todo $\varphi \in X^{*}$. Mas um vetor que dá zero em todo funcional no dual só pode ser nulo, por Hahn-Banach. A fórmula de Cauchy é demonstrada de modo análogo.

O próximo teorema corresponde ao Teorema de Cauchy acima, porém no caso de curvas envolvendo conjuntos não limitados.

Escrevemos $\alpha_{r},+\gamma_{\sigma},-\gamma_{\sigma}: \mathbb{R} \rightarrow \mathbb{C}$, respectivamente por $\alpha_{r}(t)=r e^{i t},+\gamma_{\sigma}(t)=t e^{i \sigma}$ e $-\gamma_{\sigma}(t)=$ $-t e^{i \sigma}$ para denotar tais funções.

Teorema 2.15. Sejam $\Omega$ um aberto de $\mathbb{C}, \delta>0$ e $-\pi \leq \sigma_{1}<\sigma_{2} \leq \pi$. Denotamos $\Gamma=$ $\gamma_{1, \infty} \dot{+} \gamma \dot{+} \gamma_{2, \infty}$ e $\Gamma_{n}=\gamma_{1, n} \dot{+} \gamma \dot{+} \gamma_{2, n} \dot{+} \beta_{n}$ no qual

- $\gamma$ é uma curva que sai de $\delta e^{i \sigma_{2}}$ e chega em $\delta e^{i \sigma_{1}}$;

- $\gamma_{1, n}=+\left.\gamma_{\sigma_{1}}\right|_{[\delta, n]}$ e $\gamma_{2, n}=-\left.\gamma_{\sigma_{2}}\right|_{[-n,-\delta]}$;

- $\gamma_{1, \infty}=+\left.\gamma_{\sigma_{1}}\right|_{[\delta,+\infty)}$ e $\gamma_{2, \infty}=-\left.\gamma_{\sigma_{2}}\right|_{(-\infty,-\delta]}$;

- $\beta_{n}=\left.\alpha_{n}\right|_{\left[\sigma_{1}, \sigma_{2}\right]}$

Suponha que $\Gamma$ e $\Gamma_{n}$ estão em $\Omega$ e ind $\left(\Gamma_{n}, \alpha\right)=0$ para todo $\alpha \in \mathbb{C} \backslash \Omega$ e todo $n$. Se $f \in H(\Omega ; X)$ e existem $c, \epsilon>0$ tais que $\|f(z)\| \leq c\langle z\rangle^{-1-\epsilon}$ para $z \in \Omega$ então

$$
\int_{\Gamma} f=0
$$

Demonstração. Observando que

$$
\int_{\Gamma} f=\lim _{n \rightarrow \infty} \int_{\gamma_{1, n} \dot{+} \gamma \dot{+} \gamma_{2, n}} f=\lim _{n \rightarrow \infty} \int_{\Gamma_{n}} f-\int_{\beta_{n}} f
$$

Pelo Teorema 2.14, $\int_{\Gamma_{n}} f=0$, e daí

$$
\int_{\Gamma} f=-\lim _{n \rightarrow \infty} \int_{\beta_{n}} f
$$

estimando a integral do limite acima

$$
\begin{aligned}
\left\|\int_{\beta_{n}} f\right\| & \leq\left\|\int_{\sigma_{1}}^{\sigma_{2}} f\left(n e^{i t}\right) n i e^{i t} d t\right\| \\
& \leq n \int_{\sigma_{1}}^{\sigma_{2}}\left\|f\left(n e^{i t}\right)\right\| d t \\
& \leq c\left(\sigma_{2}-\sigma_{1}\right) n\left(1+n^{2}\right)^{-1 / 2-\epsilon / 2} \rightarrow 0
\end{aligned}
$$

quando $n \rightarrow \infty$.

\subsection{Cálculo funcional}

Para cada $0<\sigma<\pi$ iremos denotar o setor aberto por $\Sigma_{\sigma}=\left\{r e^{i \theta}: r>0,|\theta|<\sigma\right\}$. Para $\lambda \in \mathbb{C}$, escrevemos $\langle\lambda\rangle=\left(1+|\lambda|^{2}\right)^{1 / 2}$.

Para $\Omega \subset \mathbb{C}$ aberto denotamos por $H^{\infty}(\Omega)$ o conjunto de funções $f: \Omega \rightarrow \mathbb{C}$ holomorfas limitadas e por $\|f\|_{\infty}=\sup _{z \in \Omega}|f(z)|$, uma norma em $H^{\infty}(\Omega)$. Observamos que $H^{\infty}(\Omega)$ com esta 
norma é um espaço de Banach, já que o limite uniforme de funções holomorfas também é uma função holomorfa.

Para $0<\sigma<\pi$ denotamos por $H_{1}^{\infty}\left(\Sigma_{\sigma}\right)$ o conjunto de funções $f: \Sigma_{\sigma} \rightarrow \mathbb{C}$ holomorfas para as quais existem $c, d>0$ tais que

$$
|f(z)| \leq c\langle z\rangle^{-d}
$$

para $z \in \Sigma_{\sigma}$. O fatos relevantes sobre este espaço estão na próxima proposição.

Proposição 2.16. Seja $0<\sigma<\pi$.

1. $H_{1}^{\infty}\left(\Sigma_{\sigma}\right) \subset H^{\infty}\left(\Sigma_{\sigma}\right)$;

2. $H_{1}^{\infty}\left(\Sigma_{\sigma}\right) \cdot H^{\infty}\left(\Sigma_{\sigma}\right) \subset H_{1}^{\infty}\left(\Sigma_{\sigma}\right)$;

3. Para $\lambda \in \mathbb{C} \backslash \bar{\Sigma}_{\sigma}$ as funções $r_{\lambda}(z)=(\lambda-z)^{-1}$ estão em $H_{1}^{\infty}\left(\Sigma_{\sigma}\right)$;

4. A sequência $\rho_{n}(z)=n(n+z)^{-1}$ pertence a $H_{1}^{\infty}\left(\Sigma_{\sigma}\right)$, satisfaz $\sup _{n}\left\|\rho_{n}\right\|_{\infty}<\infty$ e converge pontualmente para 1 .

Demonstração. Os três primeiros itens são imediatos. Pelo item $3, \rho_{n} \in H_{1}^{\infty}\left(\Sigma_{\sigma}\right)$. Fixando $z \in \Sigma_{\sigma}$ também temos $\rho_{n}(z)=(1+z / n)^{-1} \rightarrow 1$ quando $n \rightarrow \infty$. Para estimar $\left\|\rho_{n}\right\|_{\infty}=\sup _{z \in \Sigma_{\sigma}}\left|\rho_{n}(z)\right|$ observe que

$$
r_{n, \sigma}=\operatorname{dist}\left(-n, \Sigma_{\sigma}\right)=\left\{\begin{array}{ll}
n & 0<\sigma<\pi / 2 \\
n \sin (\pi-\sigma) & \pi / 2<\sigma<\pi
\end{array} .\right.
$$

Assim, dado $0<\delta<r_{n, \sigma}$ temos $\Sigma_{\sigma} \subset \mathbb{C} \backslash B(-n, \delta)$. Logo

$$
\sup _{z \in \Sigma_{\sigma}}\left|\rho_{n}(z)\right| \leq \sup _{\mathbb{C} \backslash B(-n, \delta)}\left|\rho_{n}(z)\right|=n / \delta .
$$

Fazendo $\delta \rightarrow r_{n, \sigma}$ segue $\left\|\rho_{n}\right\|_{\infty} \leq \frac{n}{n \sin (\pi-\sigma)}=\frac{1}{\sin (\pi-\sigma)}$, no caso $\pi / 2<\sigma<\pi$, e $\left\|\rho_{n}\right\|_{\infty} \leq 1$ quando $0<\sigma<\pi / 2$.

Definição 2.17. Seja A um operador em $X$ fechado e densamente definido. Dizemos que $A$ é um operador setorial de ângulo $0<\omega<\pi$ se satisfaz as seguintes condiçôes:

- $\sigma(A) \subset \Sigma_{\omega}$

- Existe $M>0$ tal que

$$
\left\|(\lambda-A)^{-1}\right\| \leq M\langle\lambda\rangle^{-1}
$$

$\operatorname{para} \lambda \in \mathbb{C} \backslash \Sigma_{\omega}$.

Observamos que nesta definição operadores setoriais são inversíveis.

Proposição 2.18. Sejam $X$ e $Y$ espaços de Banach, $S$ um subconjunto de $X$ e $T: \bar{S} \rightarrow Y$ contínua. Se $T_{n} \in \mathcal{B}(X, Y)$ é uma sequência uniformemente limitada e $\lim _{n \rightarrow \infty} T_{n} x=$ Tx para todo $x \in S$. Então $\lim _{n \rightarrow \infty} T_{n} x=T x$ também é válido para $x \in \bar{S}$.

Demonstração. Denotemos $0<M=1+\sup _{n}\left\|T_{n}\right\|<\infty$. Dado $x \in \bar{S}$ considere $\left(x_{n}\right)_{n \in \mathbb{N}}$ uma sequência em $S$ convergindo para $x$. Seja $\epsilon>0$. Como $x_{n} \rightarrow x$ e (pela continuidade de $T$ ) $T x_{n} \rightarrow T x$ podemos escolher $m$ tal que $\left\|x-x_{m}\right\|<\epsilon /(3 M)$ e $\left\|T x_{m}-T x\right\|<\epsilon / 3$. Já que $x_{m} \in S$ existe $n_{0}=n_{0}(m)$ satisfazendo $\left\|T_{n} x_{m}-T x_{m}\right\|<\epsilon / 3$ para $n \geq n_{0}$. Logo

$$
\begin{aligned}
\left\|T_{n} x-T x\right\| & \leq\left\|T_{n}\left(x-x_{m}\right)\right\|+\left\|T_{n} x_{m}-T x_{m}\right\|+\left\|T x_{m}-T x\right\| \\
& \leq M\left\|x-x_{m}\right\|+\left\|T_{n} x_{m}-T x_{m}\right\|+\left\|T x_{m}-T x\right\| \\
& <\epsilon / 3+\epsilon / 3+\epsilon / 3=\epsilon
\end{aligned}
$$

sempre que $n \geq n_{0}$. 
Se $A: D(A) \subset X \rightarrow X$ é um operador linear, lembramos que as potências $A^{n}$ são os operadores definidos por

- $D\left(A^{0}\right)=X$ e $A^{0}=I$.

- $D\left(A^{n}\right)=\left\{x \in D\left(A^{n-1}\right): A^{n-1} x \in D(A)\right\}$ e $A^{n} x=A A^{n-1} x$, se $n \geq 1$.

Proposição 2.19. Se A é um operador setorial, então $D\left(A^{n}\right)$ é denso em $X$.

Demonstração. Para $m \in \mathbb{N}(m \geq 1)$ escreva $R_{m}=(m+A)^{-1}$. Provaremos primeiro a seguinte identidade

$$
m R_{m}^{p} x=R_{m}^{p-1} x-R_{m}^{p} A x, \quad x \in D(A)
$$

para cada $p \in \mathbb{N}$, onde definimos $R_{m}^{0}=1$. O caso $p=1$ segue dos cálculos

$$
\begin{aligned}
m(m+A)^{-1} x & =(m+A-A)(m+A)^{-1} x \\
& =x-A(m+A)^{-1} x \\
& =x-(m+A)^{-1} A x
\end{aligned}
$$

onde na última igualdade usamos que $x \in D(A)$. Para $p \in \mathbb{N}$ temos

$$
\begin{aligned}
m R_{m}^{p} x & =m R_{m} R_{m}^{p-1} x \\
& =R_{m}^{p-1} x-R_{m} A R_{m}^{p-1} x \quad(\text { caso } p=1) \\
& =R_{m}^{p-1} x R_{m}^{p} A x \quad\left(A \text { e } R_{m}^{p-1} \text { comutam }\right)
\end{aligned}
$$

Em segundo lugar, partindo da hipótese que $A$ é setorial chegamos, por indução, na seguinte desigualdade

$$
\left\|R_{m}^{p}\right\| \leq M^{p}\langle m\rangle^{-p}, \quad p \in \mathbb{N}
$$

Também por indução segue de (2.1) e (2.2) que

$$
\lim _{m \rightarrow \infty} m^{p} R_{m}^{p} x=x
$$

para todo $x \in D(A)$ e $p \in \mathbb{N}$. De fato, multiplicando (2.1) por $m^{p-1}$ temos

$$
m^{p} R_{m}^{p} x=m^{p-1} R_{m}^{p-1} x-m^{p-1} R_{m}^{p} A x .
$$

Notando que $m^{p-1} R_{m}^{p} A x=m^{p-1}\langle m\rangle^{-p}\langle m\rangle^{p} R_{m}^{p} A x \rightarrow 0$ quando $m \rightarrow \infty$, verificamos o caso $p=1$ utilizando que $R_{m}^{0}=1$ e os demais casos via hipótese de indução.

Como $\left\|m^{p} R_{m}^{p}\right\| \leq C_{p}$ (por (2.2)) e $D(A)$ é denso podemos concluir, pela Proposição 2.18, que (2.3) é válido para todo $x \in X$.

Já que $y_{m}^{p}=m^{p} R_{m}^{p} x \in D\left(A^{p}\right)$ para todo $m, p \in \mathbb{N}$ e $x \in X$, mostramos acima que dado qualquer $x \in X$ a sequência $y_{m}^{p}$ pertence a $D\left(A^{p}\right)$ e converge para $x$. Logo $D\left(A^{p}\right)$ é denso em $X$.

Dados $0<\sigma<\pi$ e $r>0$ vamos denotar a curva $\Gamma_{\sigma, r}=\partial\left(\Sigma_{\sigma} \backslash\{|\lambda| \leq r\}\right)$ orientada positivamente, isto é, percorremos a curva no sentido que faz a parte imaginária diminuir.

Proposição 2.20. Seja $A$ um operador setorial de ângulo $0<\omega<\pi e \omega<\sigma<\pi$. Para cada $f \in H_{1}^{\infty}\left(\Sigma_{\sigma}\right)$, a seguinte integral existe e define um operador em $\mathcal{B}(X)$

$$
\Phi_{A}(f) \doteq \frac{1}{2 \pi i} \int_{\Gamma_{\sigma_{1}, r}} f(\lambda)(\lambda-A)^{-1} d \lambda
$$

onde $\omega<\sigma_{1}<\sigma$ e $0<r<\left\|A^{-1}\right\|^{-1}$ não altera o valor da integral. 
Demonstraçãa. Denote $\Sigma_{\alpha, \beta}=\left\{r e^{i \theta}: r>0, \alpha<\theta<\beta\right\}$. Fixe $f \in H_{1}^{\infty}\left(\Sigma_{\sigma}\right)$. Como $g: \Sigma_{\sigma} \cap$ $\left[\left(\mathbb{C} \backslash \Sigma_{\omega}\right) \cup\left\{|\lambda| \leq\left\|A^{-1}\right\|^{-1}\right\}\right] \rightarrow \mathcal{B}(X)$ dada por $g(\lambda)=f(\lambda)(\lambda-A)^{-1}$ é contínua e satisfaz $\|g\|=|f(\lambda)|\left\|(\lambda-A)^{-1}\right\| \leq c M\langle\lambda\rangle^{-1-d}$ temos que a integral

$$
I\left(\Gamma_{\sigma_{1}, r_{1}}\right)=\frac{1}{2 \pi i} \int_{\Gamma_{\sigma_{1}, r_{1}}} g(\lambda) d \lambda
$$

é um elemento bem definido de $\mathcal{B}(X)$ para cada $\omega<\sigma_{1}<\sigma$ e $0<r_{1}<\left\|A^{-1}\right\|^{-1}$.

Para verificar a independência com relação à $\sigma_{1}$ e $r_{1}$ note que integrar sobre $\Gamma_{\sigma_{1}, r_{1}} \dot{+}\left(-\Gamma_{\sigma_{1}, r_{2}}\right)$ equivale a integrar sobre o ciclo $\partial\left(\Sigma_{\sigma} \cap\left\{r_{2}<|\lambda|<r_{1}\right\}\right)$ (supondo $0<r_{2}<r_{1}$ ). Portanto pelo Teorema 2.14 temos

$$
I\left(\Gamma_{\sigma_{1}, r_{1}} \dot{+}\left(-\Gamma_{\sigma_{1}, r_{2}}\right)\right)=0
$$

$\operatorname{logo} I\left(\Gamma_{\sigma_{1}, r_{1}}\right)=I\left(\Gamma_{\sigma_{1}, r_{2}}\right)$. Além disso, integrar sobre $\Gamma_{\sigma_{1}, r_{1}} \dot{+}\left(-\Gamma_{\sigma_{2}, r_{1}}\right)$ equivale a integrar sobre as curvas ilimitadas $\partial\left(\Sigma_{\sigma_{1}, \sigma_{2}} \backslash\left\{|\lambda|<r_{1}\right\}\right)$ e $\partial\left(\Sigma_{-\sigma_{2},-\sigma_{1}} \backslash\left\{|\lambda|<r_{1}\right\}\right)$ (supondo $\sigma_{1}<\sigma_{2}$ ). Pelo Teorema 2.15 cada uma destas últimas integrais é nula e, portanto, $I\left(\Gamma_{\sigma_{1}, r_{1}}\right)=I\left(\Gamma_{\sigma_{2}, r_{1}}\right)$. Assim, dados quaisquer $\sigma_{1}<\sigma_{2}$ e $r_{2}<r_{1}$ temos

$$
I\left(\Gamma_{\sigma_{1}, r_{1}}\right)=I\left(\Gamma_{\sigma_{1}, r_{2}}\right)=I\left(\Gamma_{\sigma_{2}, r_{2}}\right)
$$

Teorema 2.21. Seja $A$ um operador setorial de ângulo $0<\omega<\pi e \omega<\sigma<\pi$. A aplicação $\Phi_{A}: H_{1}^{\infty}\left(\Sigma_{\sigma}\right) \rightarrow \mathcal{B}(X)$, definida na Proposição 2.20, é linear e satisfaz

$$
\Phi_{A}(f g)=\Phi_{A}(f) \Phi_{A}(g)
$$

para toda $f$ e g em $H_{1}^{\infty}\left(\Sigma_{\sigma}\right)$. Além disso, se $\mu \in \mathbb{C} \backslash \bar{\Sigma}_{\sigma}$ então

$$
\Phi_{A}\left(r_{\mu}\right)=(\mu-A)^{-1}
$$

onde $r_{\mu}(\lambda)=(\mu-\lambda)^{-1}$.

Demonstração. A linearidade é consequência direta da definição. Para provar que $\Phi_{A}$ é multiplicativa considere $\omega<\sigma_{1}<\sigma_{2}<\sigma$ e $0<r_{2}<r_{1}<\left\|A^{-1}\right\|^{-1}$ e $f, g \in H_{1}^{\infty}\left(\Sigma_{\sigma}\right)$. Pela independência da integral com relação as curvas, integrar sobre $\Gamma_{\sigma_{1}, r_{1}}$ equivale a integrar sobre $\Gamma_{\sigma_{2}, r_{2}}$. Assim, $\Phi_{A}(f) \Phi_{A}(g)$ é igual a

$$
\left(\frac{1}{2 \pi i} \int_{\Gamma_{\sigma_{2}, r_{2}}} f(\lambda)(\lambda-A)^{-1} d \lambda\right)\left(\frac{1}{2 \pi i} \int_{\Gamma_{\sigma_{1}, r_{1}}} g(\mu)(\mu-A)^{-1} d \mu\right)
$$

Reordenando as integrais iteradas temos

$$
\Phi_{A}(f) \Phi_{A}(g)=\left(\frac{1}{2 \pi i}\right)^{2} \int_{\Gamma_{\sigma_{2}, r_{2}}} \int_{\Gamma_{\sigma_{1}, r_{1}}} f(\lambda) g(\mu)(\lambda-A)^{-1}(\mu-A)^{-1} d \mu d \lambda
$$

e pela identidade do resolvente $(\lambda-A)^{-1}(\mu-A)^{-1}=(\mu-\lambda)^{-1}\left[(\lambda-A)^{-1}-(\mu-A)^{-1}\right]$ segue

$$
\begin{aligned}
& \Phi_{A}(f) \Phi_{A}(g) \\
= & \left(\frac{1}{2 \pi i}\right)^{2} \int_{\Gamma_{\sigma_{2}, r_{2}}} I_{g}(\lambda) f(\lambda)(\lambda-A)^{-1} d \lambda+\left(\frac{1}{2 \pi i}\right)^{2} \int_{\Gamma_{\sigma_{1}, r_{1}}} I_{f}(\mu) g(\mu)(\mu-A)^{-1} d \mu
\end{aligned}
$$

onde

$$
I_{g}(\lambda)=\int_{\Gamma_{\sigma_{1}, r_{1}}} \frac{g(\mu)}{\mu-\lambda} d \mu
$$


é igual a zero (pelo Teorema 2.15) para $\lambda \in \Gamma_{\sigma_{2}, r_{2}} \mathrm{e}$

$$
I_{f}(\mu)=\int_{\Gamma_{\sigma_{2}, r_{2}}} \frac{f(\lambda)}{\lambda-\mu} d \lambda
$$

é igual a $2 \pi i f(\mu)$ para $\mu \in \Gamma_{\sigma_{1}, r_{1}}$. Portanto

$$
\Phi_{A}(f) \Phi_{A}(g)=\frac{1}{2 \pi i} \int_{\Gamma_{\sigma_{1}, r_{1}}} f(\mu) g(\mu)(\mu-A)^{-1} d \mu=\Phi_{A}(f g)
$$

Para finalizar calculamos

$$
\begin{aligned}
\Phi_{A}\left(r_{\mu}\right) & =\frac{1}{2 \pi i} \int_{\Gamma_{\sigma_{1}, r_{1}}} \frac{1}{\mu-\lambda}(\lambda-A)^{-1} d \lambda \\
& =\frac{1}{2 \pi i} \int_{-\Gamma_{\sigma_{1}, r_{1}}} \frac{1}{\lambda-\mu}(\lambda-A)^{-1} d \lambda \\
& =(\mu-A)^{-1}
\end{aligned}
$$

onde a última igualdade vale pois o operador resolvente é holomorfo no interior de $-\Gamma_{\sigma_{1}, r_{1}}$.

Definição 2.22. Seja $A$ um operador de ângulo $0<\omega<\pi$. Dizemos que A possui cálculo $H^{\infty}$ limitado de ângulo $\sigma$ (ou apenas $A$ possui cálculo $H^{\infty}$ ) se $\Phi_{A}$ possuir uma extensão $\bar{\Phi}_{A}: H^{\infty}\left(\Sigma_{\sigma}\right) \rightarrow$ $\mathcal{B}(X)$ linear, multiplicativa e continua. Em particular existe $C_{\sigma}>0$ com

$$
\left\|\bar{\Phi}_{A}(f)\right\| \leq C_{\sigma}\|f\|_{\infty}
$$

para toda $f \in H^{\infty}\left(\Sigma_{\sigma}\right)$.

Quando $A$ possui cálculo $H^{\infty}$ a extensão na definição anterior é única. Para ver isso suponha que $\Psi_{1}$ e $\Psi_{2}$ são extensões de $\Phi_{A}$ como na Definição 2.22. No Teorema 2.21 vimos que $\Phi_{A}\left((1+z)^{-1}\right)=$ $(1+A)^{-1}$ e assim

$$
x=\Phi_{A}\left((1+z)^{-1}\right)(1+A) x
$$

para todo $x \in D(A)$. Além disso, para $f \in H^{\infty}$ e $x \in D(A)$, temos

$$
\begin{aligned}
\Psi_{1}(f) x & =\Psi_{1}(f) \Phi_{A}\left((1+z)^{-1}\right)(1+A) x \\
& =\Psi_{1}\left(f(1+z)^{-1}\right)(1+A) x \\
& =\Phi_{A}\left(f(1+z)^{-1}\right)(1+A) x
\end{aligned}
$$

e do mesmo modo

$$
\Psi_{2}(f) x=\Phi_{A}\left(f(1+z)^{-1}\right)(1+A) x .
$$

(Na demonstração do Teorema 2.23 adiante usaremos esta mesma expressão para estender o cálculo). Logo $\Psi_{1}(f) x=\Psi_{2}(f) x$ para todo $x \in D(A)$. Como $D(A)$ é denso em $X$ e $\Phi_{1}(f), \Phi_{2}(f) \in \mathcal{B}(X)$ concluímos que $\Psi_{1}(f)=\Psi_{2}(f)$.

Teorema 2.23. Seja A um operador setorial e suponha que existe $M>0$ tal que

$$
\left\|\Phi_{A}(f)\right\| \leq M\|f\|_{\infty}
$$

para toda $f \in H_{1}^{\infty}\left(\Sigma_{\sigma}\right)$. Então A possui cálculo $H^{\infty}$ com ângulo $\sigma$.

Demonstração. Dada $f \in H^{\infty}\left(\Sigma_{\sigma}\right)$ defina

$$
\bar{\Phi}_{A}(f) x=\Phi_{A}\left(f \rho_{1}\right)(1+A) x
$$


para $x \in D(A)$, lembrando que $\rho_{1}(z)=(1+z)^{-1}$ e $f \rho_{1} \in H_{1}^{\infty}\left(\Sigma_{\sigma}\right)$. Iremos provar que $\bar{\Phi}_{A}(f)$ tem extensão única a um operador em $\mathcal{B}(X)$. Considere $\rho_{n}(z)=(1+z / n)^{-1}$. Se $x \in D\left(A^{2}\right)$ então

$$
\begin{aligned}
\bar{\Phi}_{A}(f) x & =\Phi_{A}\left(f \rho_{1}\right)(1+A) x \\
& =\Phi_{A}\left(f \rho_{1}\right) \Phi_{A}\left(\rho_{n}\right)\left(1+n^{-1} A\right)(1+A) x \\
& =\Phi_{A}\left(f \rho_{n}\right) \Phi_{A}\left(\rho_{1}\right)(1+A)\left(1+n^{-1} A\right) x \\
& =\Phi_{A}\left(f \rho_{n}\right)\left(1+n^{-1} A\right) x
\end{aligned}
$$

onde usamos $\Phi_{A}\left(\rho_{n}\right)=\left(1+n^{-1} A\right)^{-1}$ e o fato que $(\lambda+A)$ e $(\mu+A)$ comutam para quaisquer $\lambda, \mu \in \mathbb{C}$. Logo, se $x \in D\left(A^{2}\right)$

$$
\left\|\bar{\Phi}_{A}(f) x\right\|=\left\|\Phi_{A}\left(f \rho_{n}\right)\left(1+n^{-1} A\right) x\right\| \leq M L\|f\|_{\infty}\left\|\left(1+n^{-1} A\right) x\right\|
$$

onde $L=\sup _{n}\left\|\rho_{n}\right\|_{\infty}<\infty$. Notando que $\lim _{n \rightarrow \infty}\left(1+n^{-1} A\right) x=x$, temos

$$
\left\|\bar{\Phi}_{A}(f) x\right\| \leq M L\|f\|_{\infty}\|x\|
$$

para todo $x \in D\left(A^{2}\right)$. Como $D\left(A^{2}\right)$ é denso em $X$ podemos concluir que $\bar{\Phi}_{A}(f)$ tem uma única extensão em $\mathcal{B}(X)$ e vale

$$
\left\|\bar{\Phi}_{A}(f)\right\| \leq M L\|f\|_{\infty}
$$

para todo $f \in H^{\infty}\left(\Sigma_{\sigma}\right)$. Segue facilmente da definição que $\bar{\Phi}_{A}$ é linear e estende $\Phi_{A}$. Para verificar que $\bar{\Phi}_{A}$ é multiplicativa tome $x \in D\left(A^{2}\right)$ e note

$$
\begin{aligned}
& \bar{\Phi}_{A}(f g) x \\
& =\Phi_{A}\left(f g(1+z)^{-2}\right)(1+A)^{-2} x \\
& =\Phi_{A}\left(f(1+z)^{-1}\right) \Phi_{A}\left(g(1+z)^{-1}\right)(1+A)^{-2} x \\
& =\bar{\Phi}_{A}(f) \bar{\Phi}_{A}(g) x
\end{aligned}
$$




\section{Capítulo 3}

\section{Operadores Pseudodiferenciais}

Iniciamos este capítulo enunciando definições e resultados sobre a classe de símbolos $S_{\rho, \delta}^{m}$ e os operadores pseudodiferenciais. Utilizaremos uma apresentação clássica que de forma semelhante pode ser encontrada no livro do Kumano-Go [Kg81]. Nesta parte, os conceitos elementares mais importantes são a expansão assintótica do símbolo da composição e a extensão dos operadores ao espaço das distribuições temperadas via o operador adjunto.

Em seguida, apresentamos dois resultados clássicos da teoria. O primeiro diz que a cada símbolo em $S_{1, \delta}^{0}(\delta<1)$ associamos continuamente a extensão em $L^{p}(1<p<\infty)$ do operador pseudodiferencial correspondente. Este fato vai permitir aplicarmos o cálculo funcional do Capítulo 2 aos espaços $L^{p}$. O segundo resultado é uma afirmação semelhante nos espaços de Sobolev $W_{p}^{s}$ e símbolos de ordem $m \in \mathbb{R}$ arbitrária. Uma aplicação deste último resultado aparece na demonstração de que a composição de símbolos é contínua.

Finalizamos discutindo álgebras de Fréchet e mostramos como deduzir duas propriedades importantes, a respeito dos elementos inversíveis, da álgebra $S_{\rho, \delta}^{0}$ a partir de um teorema obtido por Beals.

\subsection{Preliminares}

O espaço de Schwartz $\mathcal{S}$ é formado pelas funções $u \in C^{\infty}\left(\mathbb{R}^{n}\right)$ tais que $p_{\alpha, \beta}(u)=\sup _{x \in \mathbb{R}^{n}}\left|x^{\alpha} \partial^{\beta} u(x)\right|<$ $\infty$, para todo $\alpha, \beta \in \mathbb{N}_{0}^{n}$. Podemos mostrar que com a família de seminormas $p_{\alpha, \beta}, \mathcal{S}$ é um espaço de Frechét. Indicamos a seguir algumas propriedades importantes de $\mathcal{S}$.

Proposição 3.1. As seguintes afirmações são verdadeiras:

- A inclusão $\mathcal{S} \hookrightarrow L^{p}$ é continua.

- O conjunto $\mathcal{S}$ é denso em $L^{p}$, se $1 \leq p<\infty$.

Denotamos por $\mathcal{S}^{\prime}$ o espaço das distribuições temperadas. Recordamos que uma distribuição temperada é um funcional linear contínuo em $\mathcal{S}$. Podemos verificar que $\varphi \in \mathcal{S}^{\prime}$ através da condição

$$
u_{n} \stackrel{\mathcal{S}}{\longrightarrow} 0 \Rightarrow \varphi\left(u_{n}\right) \stackrel{\mathbb{C}}{\longrightarrow} 0
$$

Para $f: \mathbb{R}^{n} \rightarrow \mathbb{C}$ mensurável denote

$$
T_{f}(\varphi)=\int_{\mathbb{R}^{n}} f(x) \varphi(x) d x
$$

$\operatorname{com} \varphi \in \mathcal{S}$. Quando a integral está bem definida e define um funcional linear $T_{f}: S \rightarrow \mathbb{C}$ contínuo, ou seja $T_{f} \in \mathcal{S}^{\prime}$, identificaremos a função $f$ com a distribuição $T_{f}$. Por exemplo, se $f \in L^{p}$ então $T_{f} \in \mathcal{S}^{\prime}$ 
Diremos que $\varphi_{n} \in \mathcal{S}^{\prime}$ converge para $\varphi \in \mathcal{S}^{\prime}$ se

$$
\varphi_{n}(u) \stackrel{\mathbb{C}}{\longrightarrow} \varphi(u)
$$

para todo $u \in \mathcal{S}$ e denotamos $\varphi_{n} \stackrel{\mathcal{S}^{\prime}}{\longrightarrow} \varphi$. Pela identificacão acima vemos que a inclusão $L^{p} \hookrightarrow \mathcal{S}^{\prime}$ é contínua, isto é

$$
u_{n} \stackrel{L^{p}}{\longrightarrow} u \Rightarrow T_{u_{n}} \stackrel{\mathcal{S}^{\prime}}{\longrightarrow} T_{u}
$$

De fato, basta aplicar a desigualdade de Hölder: $\left|T_{u}(\varphi)\right| \leq\|u\|_{p}\|\varphi\|_{q} \operatorname{com} \frac{1}{p}+\frac{1}{q}=1$.

Seja $u \in L^{1}\left(\mathbb{R}^{n}\right)$. A transformada de Fourier de $u$ é definida por

$$
\mathcal{F} u(\xi)=\hat{u}(\xi)=\int_{\mathbb{R}^{n}} e^{-i x \cdot \xi} u(x) d x
$$

para cada $\xi \in \mathbb{R}^{n}$. Aqui usamos a notação $x . \xi=x_{1} \xi_{1}+\ldots+x_{n} \xi_{n}$.

Por conveniência enunciamos os resultados clássicos sobre a transformada de Fourier.

Proposição 3.2. As seguintes afirmações são verdadeiras:

- Para cada $u \in \mathcal{S}$ temos $\mathcal{F} u \in \mathcal{S}$ e a aplicação $\mathcal{F}: \mathcal{S} \rightarrow \mathcal{S}$ é contínua.

- $\mathcal{F}\left(D^{\alpha} u\right)(\xi)=\xi^{\alpha} \mathcal{F} u(\xi)$.

- $D^{\alpha}(\mathcal{F} u)(\xi)=\mathcal{F}\left((-x)^{\alpha} u\right)(\xi)$.

- A transformada de Fourier $\mathcal{F}: \mathcal{S} \rightarrow \mathcal{S}$ é bijetora com a seguinte inversa

$$
\mathcal{F}^{-1} v(x)=(2 \pi)^{-n} \int_{\mathbb{R}^{n}} e^{i x \cdot \xi} v(\xi) d \xi
$$

\subsection{Operadores Pseudodiferenciais}

Uma função $a: \mathbb{R}^{n} \times \mathbb{R}^{n} \rightarrow \mathbb{C}$ de classe $C^{\infty}$ é um símbolo de ordem $m$ e parâmetros $\rho$ e $\delta$ se, para todo $\alpha, \beta \in \mathbb{N}_{0}^{n}$, existe $C_{\alpha, \beta}>0 \mathrm{com}$

$$
\left|D_{\xi}^{\alpha} D_{x}^{\beta} a(x, \xi)\right| \leq C_{\alpha, \beta}\langle\xi\rangle^{m-\rho|\alpha|+\delta|\beta|}
$$

para todo $x, \xi \in \mathbb{R}^{n}$. Denotamos o conjunto formado por estes símbolos de $S_{\rho, \delta}^{m}$. Também usamos as seguintes notações: $S^{-\infty}=\cap_{m \in \mathbb{R}} S_{1,0}^{m}$ e $S_{\rho, \delta}^{\infty}=\cup_{m \in \mathbb{R}} S_{\rho, \delta}^{m}$.

Se $a \in S_{\rho, \delta}^{m}$ então

$$
q_{\alpha, \beta}(a)=\sup _{x, \xi}\left|D_{\xi}^{\alpha} D_{x}^{\beta} a(x, \xi)\right|\langle\xi\rangle^{-m+\rho|\alpha|-\delta|\beta|}
$$

define uma seminorma em $S_{\rho, \delta}^{m}$ que o torna um espaço de Fréchet. Uma base de vizinhanças da origem é formada por conjuntos da forma

$$
\cap_{j=1}^{k}\left\{a \in S_{\rho, \delta}^{m} \mid q_{\alpha_{j}, \beta_{j}}(a)<\epsilon\right\}
$$

onde $\alpha_{j}, \beta_{j} \in \mathbb{N}_{0}^{n}, \epsilon>0$ e $k \in \mathbb{N}$. As vezes é conveniente usar a família de seminormas

$$
q_{n}(a)=\max _{|\alpha+\beta| \leq n} q_{\alpha, \beta}(a)
$$

que satisfaz $q_{n} \leq q_{n+1}$ e gera a mesma topologia.

Proposição 3.3. As seguintes aplicações estão bem definidas e são continuas:

- $S_{\rho, \delta}^{m_{1}} \hookrightarrow S_{\rho, \delta}^{m_{2}}$, se $m_{1} \leq m_{2}$. 
- $a \in S_{\rho, \delta}^{m} \mapsto D_{\xi}^{\alpha} D_{x}^{\beta} a \in S_{\rho, \delta}^{m-\rho|\alpha|+\delta|\beta|}$.

- $(a, b) \in S_{\rho, \delta}^{m_{1}} \times S_{\rho, \delta}^{m_{2}} \mapsto a+b \in S_{\rho, \delta}^{\max \left\{m_{1}, m_{2}\right\}}$ (consequência do primeiro item).

- $(a, b) \in S_{\rho, \delta}^{m_{1}} \times S_{\rho, \delta}^{m_{2}} \mapsto a b \in S_{\rho, \delta}^{m_{1}+m_{2}}$.

Dado $a$ um símbolo, definimos para cada $u \in \mathcal{S}$ e $x \in \mathbb{R}^{n}$

$$
o p[a] u(x)=(2 \pi)^{-n} \int_{\mathbb{R}^{n}} e^{i x \cdot \xi} a(x, \xi) \hat{u}(\xi) d \xi .
$$

Podemos verificar que a função op $[a] u: \mathbb{R}^{n} \rightarrow \mathbb{C}$ está em $\mathcal{S}$ e que op $[a]: \mathcal{S} \rightarrow \mathcal{S}$ é contínua na topologia usual de $\mathcal{S}$. Além disso, o operador op $[a]$ é unicamente determinado pelo seu símbolo.

Enunciamos a seguir os resultados centrais da teoria dos operadores pseudodiferenciais. Assumiremos sempre $0 \leq \delta<\rho \leq 1$.

Definição 3.4. Seja $a \in S_{\rho, \delta}^{m}$. Dizemos que uma sequência de símbolos $a_{j} \in S_{\rho, \delta}^{m_{j}}, j \geq 0$, é uma expansão assintótica para a, se $m_{j}$ é uma sequência decrescente de números reais com $\lim _{j \rightarrow \infty} m_{j}=$ $-\infty$ e, para todo inteiro $N \geq 1$,

$$
a-\sum_{j=0}^{N-1} a_{j} \in S_{\rho, \delta}^{m_{N}} .
$$

Neste caso, escrevemos $a \sim \sum_{j=0}^{\infty} a_{j}$.

Note que, embora $d_{N}=a-\sum_{j=0}^{N-1} a_{j} \in S_{\rho, \delta}^{m_{N}}$, o símbolo $d_{N+1}=d_{N}-a_{N} \in S_{\rho, \delta}^{m_{N+1}}$ está num espaço de ordem menor do que $d_{N}$ e $a_{N}$ visto que $S_{\rho, \delta}^{m_{N+1}} \subset S_{\rho, \delta}^{m_{N}}$.

Como já mencionamos, um operador pseudodiferencial é um operador linear de $\mathcal{S}$ nele mesmo. Sendo a composição de dois operadores em $\mathcal{S}$ também um operador em $\mathcal{S}$, podemos nos perguntar se a classe de operadores pseudodiferenciais é preservada quando compomos dois elementos desta classe. A próxima proposição dá uma resposta a esta pergunta e ainda fornece uma expansão assintótica para o símbolo da composição.

Proposição 3.5. Sejam $a \in S_{\rho, \delta}^{m_{1}}$ e $b \in S_{\rho, \delta}^{m_{2}}$. Então existe $c \in S_{\rho, \delta}^{m_{1}+m_{2}}$ tal que op $[c]=o p[a] \circ o p[b]$ possuindo expansão assintótica

$$
c \sim \sum_{\alpha} \frac{(-i)^{|\alpha|}}{\alpha !} \partial_{\xi}^{\alpha} a \partial_{x}^{\alpha} b .
$$

Dadas duas funções $u$ e $v$ escrevemos $(u, v)=\int_{\mathbb{R}^{n}} u(x) \overline{v(x)} d x$.

Seja $A: \mathcal{S} \rightarrow \mathcal{S}$ linear. Um operador linear $A^{*}: \mathcal{S} \rightarrow \mathcal{S}$ é o adjunto formal de $A$ se para todo $u$ e $v$ em $\mathcal{S}$ temos

$$
(A u, v)=\left(u, A^{*} v\right)
$$

Note que $A^{*}$ é único. De fato se $A_{1}$ e $A_{2}$ são adjuntos de $A$ e $v \in \mathcal{S}$ é fixado, então

$$
\left(u, A_{1} v\right)=(A u, v)=\left(u, A_{2} v\right)
$$

o que implica $\left(u, A_{1} v-A_{2} v\right)=0$ para todo $u \in \mathcal{S}$. Logo $A_{1} v=A_{2} v$.

Vamos ver como usar um adjunto para estender o operador $A$ para $\mathcal{S}^{\prime}$. Suponha que $A$ possua um adjunto $A^{*}: \mathcal{S} \rightarrow \mathcal{S}$ contínuo. Dado $u \in \mathcal{S}^{\prime}$ defina

$$
\langle B u, v\rangle=\left\langle u, \overline{A^{*} \bar{v}}\right\rangle .
$$

Não é difícil mostrar que a aplicação $v \in \mathcal{S} \rightarrow \overline{A^{*} \bar{v}} \in \mathcal{S}$ é linear e contínua. Sendo assim $B u \in \mathcal{S}^{\prime}$ e $B: \mathcal{S}^{\prime} \rightarrow \mathcal{S}^{\prime}$ é linear. Para verificar que $B$ estende $A$ observe que

$$
\langle A u, v\rangle=(A u, \bar{v})=\left(u, A^{*} \bar{v}\right)=\left\langle u, \overline{A^{*} \bar{v}}\right\rangle
$$


vale para qualquer $u, v \in \mathcal{S}$.

A próxima proposição diz que o adjunto formal de um pseudo ainda é um pseudo e fornece uma expansão assintótica para o símbolo de seu adjunto. Com isto, podemos estender um pseudo ao espaço das distribuições temperadas como foi feito acima, dado que já sabemos que um pseudo é um operador linear contínuo em $\mathcal{S}$.

Proposição 3.6. Seja $a \in S_{\rho, \delta}^{m}$. Então existe $a^{*} \in S_{\rho, \delta}^{m}$ tal que op $\left[a^{*}\right]$ é o adjunto formal de op $[a]$ possuindo expansão assintótica

$$
a^{*} \sim \sum_{\alpha} \frac{(-i)^{|\alpha|}}{\alpha !} \partial_{\xi}^{\alpha} \partial_{x}^{\alpha} \bar{a}
$$

Como $L^{p} \subset \mathcal{S}^{\prime}$ podemos considerar pseudos definidos em $L^{p}$. Para operadores de ordem zero temos o seguinte resultado clássico [Fef73].

Teorema 3.7. Seja $1<p<\infty$ e $0 \leq \delta<1$. Se $a \in S_{1, \delta}^{0}$ então op $[a] \in \mathcal{B}\left(L^{p}\right)$. Temos ainda que op : $S_{1, \delta}^{0} \rightarrow \mathcal{B}\left(L^{p}\right)$ é contínuo. No caso $p=2$, vale o mesmo para $0 \leq \delta<\rho \leq 1$ arbitrário.

Um resultado para operadores de ordem arbitrária será enunciado. Com auxílio dos operadores pseudodiferenciais vamos definir os espaços de Sobolev em $L^{p}$.

Para $s \in \mathbb{R}$ denotamos $J^{s}$ o operador com símbolo $a(x, \xi)=\langle\xi\rangle^{s}$.

Definição 3.8. Seja $1<p<\infty$ es $\in \mathbb{R}$. Definimos

$$
W_{p}^{s}=\left\{u \in \mathcal{S}^{\prime}: J^{s} u \in L^{p}\right\}
$$

Notamos que $W_{p}^{s}$ é um subespaço vetorial de $\mathcal{S}^{\prime}$ e que

$$
\|u\|_{s, p}=\left\|J^{s} u\right\|_{L^{p}}
$$

define uma norma em $W_{p}^{s}$. O par $\left(W_{p}^{s},\|.\|_{s, p}\right)$ será chamado espaço de Sobolev.

Teorema 3.9. O espaço de Sobolev é um espaço de Banach e $\mathcal{S}$ é um subconjunto denso de $W_{p}^{s}$.

Teorema 3.10. Seja $1<p<\infty, 0 \leq \delta<1$ e $m, s \in \mathbb{R}$. Se $a \in S_{1, \delta}^{m}$ então op $[a]: W_{p}^{s} \rightarrow W_{p}^{s-m}$ é contínuo. Temos ainda que op $: S_{1, \delta}^{m} \rightarrow \mathcal{B}\left(W_{p}^{s}, W_{p}^{s-m}\right)$ é contínuo. No caso $p=2$, vale o mesmo para $0 \leq \delta<\rho \leq 1$ arbitrário.

\section{3 Álgebra dos símbolos}

Nesta seção discutimos alguns aspectos da álgebra dos símbolos $S_{\rho, \delta}^{\infty}$ e da álgebra $S_{\rho, \delta}^{0}$. Começamos esclarecendo a noção abstrata de álgebra.

Definição 3.11. Uma álgebra $A$ é um espaço vetorial sobre um corpo $\mathbb{K}$ equipado com uma função $m: A \times A \rightarrow A$, chamada multiplicação (denotamos $m(a, b)=a b$ ), satisfazendo

- $(a b) c=a(b c)$

- $\alpha(a b)=(\alpha a) b=a(\alpha b)$

- $c(a+b)=c a+c b e(a+b) c=a c+b c$

para quaisquer $a, b, c \in A$ e $\alpha \in \mathbb{K}$. Vamos assumir que $m$ possui um elemento neutro $1 \in A$, isto é,

$$
a 1=1 a=a
$$

para todo $a \in A$. 
Observamos que a operação de multiplicação pode ser não comutativa, ou seja, pode haver $a b \neq b a$.

Diremos que $a \in A$ é invertível se existir $b \in A$ tal que $a b=b a=1$. É fácil ver que tal $b$ é único. De fato, se $b_{1}, b_{2} \in A$ satisfazem $b_{1} a=1$ e $a b_{2}=1$ então

$$
b_{1}=b_{1} 1=b_{1}\left(a b_{2}\right)=\left(b_{1} a\right) b_{2}=1 b_{2}=b_{2}
$$

Vamos escrever $A^{-1}$ para o conjunto de elementos invertíveis e $a^{-1}$ para inversa de $a \in A^{-1}$ (não é díficil ver que o conjunto $A^{-1}$ com a operação de multiplicação é um grupo).

Veja que $a . b(x, \xi)=a(x, \xi) b(x, \xi)$ define uma multiplicação em $S_{\rho, \delta}^{\infty}$ e satisfaz $S_{\rho, \delta}^{m_{1}} \cdot S_{\rho, \delta}^{m_{2}} \subset$ $S_{\rho, \delta}^{m_{1}+m_{2}}$. No entanto, nosso foco será na seguinte operação.

Definição 3.12. Seja $a, b \in S_{\rho, \delta}^{\infty}$. Denotamos $a \# b$ para o símbolo da composição op $[a] \circ o p[b]$. Este símbolo está bem definido, devido à unicidade do símbolo (para a classe que estamos usando) de um operador pseudodiferencial.

Definição 3.13. Uma álgebra de Fréchet é um espaço de Fréchet junto com uma operação de multiplicação contínua que torna o espaço uma álgebra.

Teorema 3.14. A função $m(a, b)=a \# b$ é uma multiplicação em $S_{\rho, \delta}^{\infty}$ e satisfaz

$$
S_{\rho, \delta}^{m_{1}} \# S_{\rho, \delta}^{m_{2}} \subset S_{\rho, \delta}^{m_{1}+m_{2}}
$$

Além disso, \# : $S_{\rho, \delta}^{m_{1}} \times S_{\rho, \delta}^{m_{2}} \rightarrow S_{\rho, \delta}^{m_{1}+m_{2}}$ é contínua. Com esta multiplicação $S_{\rho, \delta}^{\infty}$ é uma álgebra e $S_{\rho, \delta}^{0}$ é uma álgebra de Frechét.

Demonstração. A inclusão é consequência da Proposição 3.5. É simples mostrar que $m$ é uma multiplicação. Por exemplo para argumentar que $m$ é associativa, sejam $a, b$ e $c$ em $S_{\rho, \delta}^{\infty}$. Então

$$
o p[(a \# b) \# c]=o p[a \# b] \circ o p[c]=(o p[a] \circ o p[b]) \circ o p[c]
$$

e

$$
o p[a \#(b \# c)]=o p[a] \circ o p[b \# c]=o p[a] \circ(o p[b] \circ o p[c]) .
$$

Como a composição de funções é associativa, segue que $o p[(a \# b) \# c]=o p[a \#(b \# c)]$. Logo, pela unicidade dos símbolos, $(a \# b) \# c=a \#(b \# c)$. As demais propriedades seguem o mesmo racíocinio.

Vamos mostrar que $m$ é contínua. Notamos primeiro que como os espaços $S_{\rho, \delta}^{m}(m \in \mathbb{R})$ são Fréchet e a multiplicação é bilinear basta provar que $m$ é separadamente contínua, isto é, basta que as aplicações lineares $a \in S_{\rho, \delta}^{m_{1}} \mapsto a \# b_{0} \in S_{\rho, \delta}^{m_{1}+m_{2}}$ e $b \in S_{\rho, \delta}^{m_{2}} \mapsto a_{0} \# b \in S_{\rho, \delta}^{m_{1}+m_{2}}$ sejam contínuas para todo $a_{0} \in S_{\rho, \delta}^{m_{1}}$ e $b_{0} \in S_{\rho, \delta}^{m_{2}}$ fixados.

Pelo Teorema do gráfico fechado, a continuidade da aplicação $m_{a_{0}}: S_{\rho, \delta}^{m_{2}} \rightarrow S_{\rho, \delta}^{m_{1}+m_{2}}, m_{a_{0}}(b)=$ $a_{0} \# b$ (multiplicação à esquerda por $a_{0}$ ) é equivalente ao gráfico de $m_{a_{0}}$ ser fechado. Para mostrar que $m_{a_{0}}$ tem gráfico fechado, sejam $b, b_{n} \in S_{\rho, \delta}^{m_{2}}$ e $c \in S_{\rho, \delta}^{m_{1}+m_{2}}$ tais que $b_{n} \rightarrow b$ em $S_{\rho, \delta}^{m_{2}}$ e $a_{0} \# b_{n} \rightarrow c$ em $S_{\rho, \delta}^{m_{1}+m_{2}}$. Pela continuidade de op no Teorema 3.10, temos

1. $o p\left[b_{n}\right] \rightarrow o p[b]$ em $\mathcal{B}\left(W^{m_{1}+m_{2}}, W^{m_{1}}\right)$.

2. $o p\left[a_{0}\right] \circ o p\left[b_{n}\right]=o p\left[a_{0} \# b_{n}\right] \rightarrow o p[c]$ em $\mathcal{B}\left(W^{m_{1}+m_{2}}, W^{0}\right)$.

Ainda, como op $\left[a_{0}\right] \in \mathcal{B}\left(W^{m_{1}}, W^{0}\right)$ e pelo item 1 ,

3. $o p\left[a_{0}\right] \circ o p\left[b_{n}\right] \rightarrow o p\left[a_{0}\right] \circ o p[b]=o p\left[a_{0} \# b\right]$ em $\mathcal{B}\left(W^{m_{1}+m_{2}}, W^{0}\right)$.

Dos itens 2 e 3 e pela unicidade do limite temos que $o p\left[a_{0} \# b\right]=o p[c]$. Logo a unicidade do símbolo garante que $a_{0} \# b=c$, mostrando que o gráfico de $m_{a_{0}}$ é fechado. A continuidade da multiplicação à direita é demonstrada de modo análogo. 


\subsection{Inversa e $\psi$-Álgebra}

O resultados completos desta seção podem ser encontrado em [Wae71]. Dados $X$ e $Y$ dois subconjuntos de uma álgebra denotamos $X Y=\{x y: x \in X, y \in Y\}$.

Definição 3.15. Seja $B$ uma álgebra. Um subconjunto $A$ de $B$ é uma subalgebra de $B$ se $A$ é um subespaço vetorial de $B$ e $A A \subset A$.

Uma subalgebra $A$ de $B$ sempre é uma álgebra munida das operações induzidas. No entanto, $A$ pode não ter unidade ou mesmo ter uma unidade diferente daquela de $B$. Se $A$ é uma subalgebra com mesma unidade então $A$ é uma álgebra com unidade e temos a seguinte relação: $A^{-1} \subset A \cap B^{-1}$. Esta inclusão pode ser própria significando que pode haver um elemento de $A$ invertível em $B$, mas com inversa fora de $A$ (logo não-invertível em $A$ ).

Definição 3.16. Seja B uma álgebra de Banach. Dizemos que A é uma $\psi$-álgebra ou $\psi$-subalgebra de $B$ se

- A é uma subalgebra de B com mesma unidade e satisfaz

$$
A^{-1}=A \cap B^{-1}
$$

- A é uma álgebra de Fréchet com uma topologia mais forte que a induzida por B, ou seja, $A \hookrightarrow B$ é contínuo.

Teorema 3.17. Se A é uma $\psi$-álgebra, então $A^{-1}$ é aberto.

Demonstração. Como $B$ é uma álgebra de Banach, já sabemos que $B^{-1}$ é aberto em $B$. Logo, sendo a topologia de $A$ mais forte, temos que $A \cap B^{-1}=A^{-1}$ é aberto em $A$.

Vamos verificar agora o seguinte: A operação de inversão de uma álgebra de Fréchet $A$ em que $A^{-1}$ é aberto é necessariamente contínua.

Proposição 3.18. Seja $(M, d)$ uma espaço métrico completo e $A \subset M$ um conjunto aberto. Existe uma métrica $d_{1}$ em A tal que

- $O$ espaço $\left(A, d_{1}\right)$ é completo.

- A métrica $d_{1}$ é equivalente à métrica induzida por d.

Demonstração. Podemos supor que $A$ é não vazio nem $M$. Escreva $B=M \backslash A$. Note que a aplicação $(t, x) \in \mathbb{R} \times M \mapsto t d(x, B) \in \mathbb{R}$ é contínua e, portanto, o conjunto $F=\{(t, x) \in \mathbb{R} \times M: t d(x, B)=$ $1\}$ é fechado em $\mathbb{R} \times M$. Como $\mathbb{R} \times M$ é completo segue que $F$ é completo. Vamos mostrar que a projeção restrita a $F$ é um homeomorfismo entre $F$ e $A$. Dado $(t, x) \in F$ temos $d(x, B)>0$ e, daí, temos $x \in A$. Isto mostra que a função projeção restrita a $F$ tem imagem em $A$, isto é, $p: F \rightarrow A$ e $p(t, x)=x$. Claro que $p$ é contínua. Definindo $q: A \rightarrow F$ por $q(x)=\left(d(x, B)^{-1}, x\right)$ temos que $q$ é contínua e $p \circ q(x)=x$ e $q \circ p(t, x)=(t, x)$. Logo $p$ é bijetora e $p^{-1}=q$.

Para finalizar defina $d_{1}$ como a métrica induzida em $A$ por $q$, isto é,

$$
d_{1}(x, y)=d_{F}(q(x), q(y))
$$

$\operatorname{Assim}\left(A, d_{1}\right)$ é completo, pois $F$ é completo, e $q:\left(A, d_{1}\right) \rightarrow\left(F, d_{F}\right)$ é uma isometria (bijetora). Disto segue que $d_{1}$ e $d$ são equivalentes.

Proposição 3.19. Seja $G$ um grupo e d uma métrica em $G$ tal que

- O espaço $(G, d)$ é completo;

- A operação do grupo é separadamente contínua; 
Então a inversão, $x \in G \mapsto x^{-1} \in G$, é contínua.

Demonstração. A demonstração pode ser encontrada em [Sch95].

Teorema 3.20. Seja A uma álgebra de Fréchet com unidade. Se $A^{-1}$ é aberto então a inversão é continua.

Demonstração. Tomando em $A^{-1}$ a métrica da Proposição 3.18, vemos que estamos nas condições da Proposição 3.19. Portanto, a inversão será contínua em ambas as métricas já que elas são equivalentes.

O seguinte resultado será usado sem demonstração [Bea77].

Teorema 3.21. $S_{\rho, \delta}^{0}$ é uma $\psi$-subalgebra de $\mathcal{B}\left(L^{2}\left(\mathbb{R}^{n}\right)\right)$.

Chamando de $G=\left(S_{\rho, \delta}^{0}\right)^{-1}$ este resultado junto com os Teoremas 3.17 e 3.20 mostram que $G$ é aberto e $a \in G \mapsto a^{-\#} \in G$ é contínua. 


\section{Capítulo 4}

\section{Cálculo $H^{\infty}$ para operadores pseudodiferenciais}

Neste capítulo demonstramos que uma classe de operadores pseudodiferenciais possui cálculo $H^{\infty}$.

Primeiro vamos esclarecer em que tipo de espaço de Banach nossos operadores estão agindo. Se $a \in S_{\rho, \delta}^{m}$ não é difícil ver que o operador op $[a]: \mathcal{S} \subset L^{p}\left(\mathbb{R}^{n}\right) \rightarrow L^{p}\left(\mathbb{R}^{n}\right)$ é fechável e podemos nos questionar se seu fecho é setorial e se possui cálculo $H^{\infty}$. De modo mais geral, podemos verificar que se $E$ é um espaço de Banach de distribuições temperadas contendo $\mathcal{S}$ e a inclusão $E \hookrightarrow \mathcal{S}^{\prime}$ é contínua, então op $[a]: \mathcal{S} \subset E \rightarrow E$ é fechável (ver Proposição 4.14). Isto permite colocar as mesmas questões anteriores para o fecho deste operador em $E$.

Para um elemento $a$ da classe de símbolos encontradas na Definição 4.1 e supondo que o espaço E satisfaz a seguinte condição

$$
\text { op } \in \mathcal{B}\left(S_{\rho, \delta}^{0}, \mathcal{B}(E)\right)
$$

iremos mostrar que o fecho de op $[a]$ possui cálculo $H^{\infty}$ (mais precisamente uma translação deste operador, ver Teorema 4.12). A condição (4.1) diz que o operador op associa continuamente símbolos de ordem zero à operadores limitados em $E$. No Teorema 3.7 do Capítulo 3 já vimos que $E=L^{p}\left(\mathbb{R}^{n}\right)$ satisfaz esta condição quando $\rho=1$ e $\delta<1$. Daremos a seguir uma ideia geral da demonstração.

Baseado na construção da parametriz dos símbolos $a-\lambda$, como encontrada nos livros do KumanoGo [Kg81] ou Wong [Won14], estabelecemos uma espécie de cálculo funcional para a na álgebra de Fréchet $S_{\rho, \delta}^{0}$ (ver Teorema 4.11). Como vimos na seção 3.4 do Capítulo 3 a álgebra $S_{\rho, \delta}^{0}$ tem um grupo de invertíveis aberto. Este fato possibilitará o uso do seguinte argumento clássico de análise: Se $A$ é uma matriz invertível e $B_{n} \rightarrow 0$ então $A+B_{n}$ é invertível para todo $n$ suficientemente grande (ver demonstração do Teorema 4.8). Por fim, a condição (4.1) permitirá passar estimativas nas seminormas de $S_{\rho, \delta}^{0}$ para norma de operadores em $\mathcal{B}(E)$, e assim, chegar a condição suficiente do Teorema 2.23 do Capítulo 2.

\subsection{Cálculo para o símbolo}

Denotamos por $M_{d}$ o conjunto das matrizes $d \times d$ complexas e $M_{d}^{-1}$ o subconjunto das matrizes invertíveis. Assim o conjunto resolvente de $a \in M_{d}$ é $\rho(a)=\left\{\lambda \in \mathbb{C}:(a-\lambda) \in M_{d}^{-1}\right\}$. Fixamos $0<\theta<\pi$ e denotamos $\Lambda=\mathbb{C} \backslash \Sigma_{\theta}$. Mais explicitamente

$$
\Lambda=\left\{r e^{i \varphi}: r \geq 0, \quad|\varphi| \geq \theta\right\}
$$

Definição 4.1. Sejam $0 \leq \delta<\rho \leq 1, m \geq 0$ e $a \in S_{\rho, \delta}^{m}$ um símbolo podendo tomar valores matriciais. Dizemos que a é um simbolo HS (Hipoelíptico Setorial) se existir c $>0$ tais que

1. Para $x, \xi \in \mathbb{R}^{n}$

$$
\Lambda \cup\{|\lambda| \leq c\} \subset \rho(a(x, \xi))
$$


No caso escalar $\rho(a(x, \xi))=\mathbb{C} \backslash\{a(x, \xi)\}$, logo a condição acima equivale a dizer que $a(x, \xi)$ não pertence ao conjunto $\Lambda \cup\{|\lambda| \leq c\}$.

2. Para todo $\alpha, \beta \in \mathbb{N}_{0}^{n}$ existe $c_{\alpha, \beta}>0$ tal que

$$
\left|\partial_{\xi}^{\alpha} \partial_{x}^{\beta} a(x, \xi)\right|\left|(a(x, \xi)-\lambda)^{-1}\right| \leq c_{\alpha, \beta}\langle\xi\rangle^{-\rho|\alpha|+\delta|\beta|}
$$

sempre que $x, \xi \in \mathbb{R}^{n}$ e $\lambda \in \Lambda$.

A seguinte notação será conveniente

$$
\Omega_{x, \xi}=\left\{z \in \Sigma_{\theta}:|z|<2|a(x, \xi)|\right\} .
$$

Proposição 4.2. Seja a um símbolo $H S$.

- A estimativa 2 da Definição 4.1 continua valendo ao trocar o conjunto $\Lambda$ por $\mathbb{C} \backslash \Omega_{x, \xi}$.

- Podemos encontrar $c_{0}>0$ tal que

$$
\left|(a(x, \xi)-\lambda)^{-1}\right| \leq c_{0}\langle\lambda\rangle^{-1}
$$

$\operatorname{para} \lambda \in \mathbb{C} \backslash \Omega_{x, \xi}$.

Demonstração. Observe primeiro que para $\lambda \in \rho(a) \backslash\{0\}$ e $|\lambda|>|a|$

$$
\left|(a-\lambda)^{-1}\right|=|\lambda|^{-1}\left|(a / \lambda-1)^{-1}\right| \leq|\lambda|^{-1} \sum_{k=0}^{\infty}|a / \lambda|^{k}=|\lambda|^{-1}(1-|a / \lambda|)^{-1}
$$

Agora seja a um símbolo HS. Dados $\alpha, \beta \in \mathbb{N}_{0}^{n}$ tomamos $c_{\alpha, \beta}>0$ da estimativa 2. Notando que $\mathbb{C} \backslash \Omega_{x, \xi}$ é a união de $\Lambda$ e $\mathbb{C} \backslash B(0,2|a(x, \xi)|)$ basta mostrar a afirmação para $|\lambda| \geq 2|a(x, \xi)|$. Neste caso $(1-|a / \lambda|)^{-1} \leq 2$. Como $a(x, \xi)$ é invertível temos ainda $|\lambda|^{-1} \leq(2|a(x, \xi)|)^{-1} \leq\left|a(x, \xi)^{-1}\right| / 2$. Logo usando a observação acima e estimativa 2 no caso $\lambda=0$

$$
\begin{aligned}
\left|\partial_{\xi}^{\alpha} \partial_{x}^{\beta} a(x, \xi)\right|\left|(a(x, \xi)-\lambda)^{-1}\right| & \leq\left|\partial_{\xi}^{\alpha} \partial_{x}^{\beta} a(x, \xi)\right||\lambda|^{-1}(1-|a(x, \xi) / \lambda|)^{-1} \\
& \leq\left|\partial_{\xi}^{\alpha} \partial_{x}^{\beta} a(x, \xi)\right|\left(\left|a(x, \xi)^{-1}\right| / 2\right) 2 \\
& =\left|\partial_{\xi}^{\alpha} \partial_{x}^{\beta} a(x, \xi)\right|\left|a(x, \xi)^{-1}\right| \\
& \leq c_{\alpha, \beta}\langle\xi\rangle^{-\rho|\alpha|+\delta|\beta|}
\end{aligned}
$$

Para mostrar a segunda afirmação veja que da identidade $a(a-\lambda)^{-1}=1+\lambda(a-\lambda)^{-1}$ segue

$$
\left|(a-\lambda)^{-1}\right| \leq|\lambda|^{-1}\left|a(a-\lambda)^{-1}-1\right| \leq|\lambda|^{-1}\left(1+c_{0,0}\right)
$$

onde $c_{0,0}$ é obtido do primeiro item fazendo $\alpha=\beta=0$. Como $a$ é invertível obtemos a desigualdade desejada.

Nosso objetivo será chegar em uma representação para inversa do símbolo $a-\lambda$ com relação ao produto \#. Para isso usaremos uma construção clássica da parametriz.

Definição 4.3. Para $(x, \xi) \in \mathbb{R}^{n} \times \mathbb{R}^{n}$ e $\lambda \in \mathbb{C} \backslash \Omega_{x, \xi}$ definimos

$$
\begin{aligned}
b_{0}(x, \xi ; \lambda) & =(a(x, \xi)-\lambda)^{-1} \\
b_{j+1}(x, \xi ; \lambda) & =-b_{0}(x, \xi ; \lambda) \sum_{\substack{|\alpha|+k=j+1, 0 \leq k \leq j}} \frac{1}{\alpha !} \partial_{\xi}^{\alpha} a(x, \xi) D_{x}^{\alpha} b_{k}(x, \xi ; \lambda)
\end{aligned}
$$

se $j \geq 0$. 
Como $b_{0}$ é $C^{\infty}$ podemos verificar que cada $b_{j}$ é $C^{\infty}$ e a recursão está bem definida. Isto significa que podemos computar previamente as derivadas de cada $b_{k}$ no somatório. Notemos que a recursão na Definição $4.3 \mathrm{em}$ geral, preserva as propriedades de $b_{0}$. Por exemplo, é fácil ver que $b_{0}$ é analítica em $\lambda$ e portanto cada $b_{j}$ também será.

Calculando as derivadas de ordem 1 de $b_{0}$ temos

$$
\partial b_{0}=b_{0} \partial a b_{0},
$$

com isto podemos calcular, por exemplo, a derivada $\partial^{2}=\partial_{\xi}^{2}$ e obter

$$
\partial^{2} b_{0}=\left(b_{0} \partial a b_{0}\right) \partial a b_{0}+b_{0}\left(\partial^{2} a\right) b_{0}+b_{0} \partial a\left(b_{0} \partial a b_{0}\right) .
$$

Por indução conseguimos mostrar que para cada $\alpha, \beta \in \mathbb{N}_{0}^{n}$ e $j \in \mathbb{N}_{0}$ existe um $r \in \mathbb{N}_{0}$ para o qual $\partial_{\xi}^{\alpha} \partial_{x}^{\beta} b_{j}$ se escreve como combinação linear de termos da forma

$$
b_{0}(x, \xi ; \lambda) \partial_{\xi}^{\alpha_{1}} \partial_{x}^{\beta_{1}} a(x, \xi) b_{0}(x, \xi ; \lambda) \ldots \partial_{\xi}^{\alpha_{r}} \partial_{x}^{\beta_{r}} a(x, \xi) b_{0}(x, \xi ; \lambda)
$$

onde $\alpha_{k}$ e $\beta_{k}$ são multi-índices em $\mathbb{N}_{0}^{n}$ tais que $\sum_{k=1}^{r}\left|\alpha_{k}\right|=|\alpha|+j$ e $\sum_{k=1}^{r}\left|\beta_{k}\right|=|\beta|+j$.

Definição 4.4. Para $(x, \xi) \in \mathbb{R}^{n} \times \mathbb{R}^{n}$ e $\lambda \in \mathbb{C} \backslash \Omega_{x, \xi}$ definimos

$$
b^{N}(x, \xi ; \lambda)=\sum_{j<N} b_{j}(x, \xi ; \lambda)
$$

e se $\lambda \in \Lambda$,

$$
r^{N}(\lambda)=(a-\lambda) \# b^{N}(\lambda)-1
$$

onde $N \geq 1$.

Apesar de ser uma simples consequência das observações anteriores a próxima proposição merece ser destacada devido à sua repetida utilização em algumas demonstrações.

Proposição 4.5. Sejam $\alpha, \beta \in \mathbb{N}_{0}^{n}$ e $j \geq 0$. Então existe $M>0$ tal que

$$
\left|\partial_{\xi}^{\alpha} \partial_{x}^{\beta} b_{j}(x, \xi ; \lambda)\right| \leq M\left|(a(x, \xi)-\lambda)^{-1}\right|\langle\xi\rangle^{-(\rho-\delta) j-\rho|\alpha|+\delta|\beta|}
$$

para todo $x, \xi \in \mathbb{R}^{n}$ e $\lambda \in \mathbb{C} \backslash \Omega_{x, \xi}$.

Demonstração. Sejam $j \geq 0$ e $r>0$ inteiros, $\alpha, \beta, \alpha_{k}, \beta_{k}$, com $1 \leq k \leq r$, multi-índices tais que $\left|\alpha_{1}\right|+\cdots+\left|\alpha_{r}\right|=j+|\alpha|$ e $\left|\beta_{1}\right|+\cdots+\left|\beta_{r}\right|=j+|\beta|$. Defina

$$
T(\lambda)=b_{0}(\lambda) \partial_{\xi}^{\alpha_{1}} \partial_{x}^{\beta_{1}} a b_{0}(\lambda) \ldots \partial_{\xi}^{\alpha_{r}} \partial_{x}^{\beta_{r}} a b_{0}(\lambda)
$$

Do primeiro item da Proposição 4.2 temos

$$
|T(\lambda)| \leq M\left|b_{0}(\lambda)\right|\langle\xi\rangle^{-\rho\left(\left|\alpha_{1}\right|+\ldots\left|\alpha_{r}\right|\right)+\delta\left(\left|\beta_{1}\right|+\ldots\left|\beta_{r}\right|\right)}=M\left|b_{0}(\lambda)\right|\langle\xi\rangle^{-j(\rho-\delta)-\rho|\alpha|+\delta|\beta|}
$$

Desta estimativa e da observação sobre $\partial_{\xi}^{\alpha} \partial_{x}^{\beta} b_{j}$ obtemos

$$
\left|\partial_{\xi}^{\alpha} \partial_{x}^{\beta} b_{j}(\lambda)\right| \leq c_{\alpha, \beta}\left|b_{0}(\lambda)\right|\langle\xi\rangle^{-j(\rho-\delta)-\rho|\alpha|+\delta|\beta|}
$$

Proposição 4.6. A função $\lambda \in \Lambda \mapsto\langle\lambda\rangle b_{j}(\lambda) \in S_{\rho, \delta}^{-j(\rho-\delta)}$ é contínua e limitada.

Demonstração. Seja $\lambda \in \Lambda$. Como pelo segundo item da Proposição 4.2 vale

$$
\left|b_{0}(\lambda)\right| \leq c_{0}\langle\lambda\rangle^{-1}
$$


concluímos da Proposição 4.5 que

$$
\left|\partial_{\xi}^{\alpha} \partial_{x}^{\beta} b_{j}(x, \xi ; \lambda)\right| \leq c_{\alpha, \beta}\langle\lambda\rangle^{-1}\langle\xi\rangle^{-j(\rho-\delta)-\rho|\alpha|+\delta|\beta|}
$$

para todo $x, \xi \in \mathbb{R}^{n}$ já que $\Lambda \subset \mathbb{C} \backslash \Omega_{x, \xi}$. Isso mostra que $b_{j}(\lambda) \in S_{\rho, \delta}^{-j(\rho-\delta)} \mathrm{e}$

$$
q_{\alpha, \beta}^{-j(\rho-\delta)}\left(b_{j}(\lambda)\right) \leq c_{\alpha, \beta}\langle\lambda\rangle^{-1}
$$

para todo $\lambda \in \Lambda$. Logo $\lambda \in \Lambda \mapsto\langle\lambda\rangle b_{j}(\lambda) \in S_{\rho, \delta}^{-j(\rho-\delta)}$ é limitada.

Vamos verificar a continuidade de $b_{0}$. Seja $\lambda_{n} \rightarrow \lambda$ uma sequência convergente em $\Lambda$. Pela identidade do resolvente

$$
b_{0}\left(\lambda_{n}\right)-b_{0}(\lambda)=\left(\lambda_{n}-\lambda\right) b_{0}\left(\lambda_{n}\right) b_{0}(\lambda)
$$

e como já mostramos que $\left\{b_{0}\left(\lambda_{n}\right): n \in \mathbb{N}_{0}\right\}$ é limitado em $S_{\rho, \delta}^{0}$ concluímos que $b_{0}\left(\lambda_{n}\right) \rightarrow b_{0}(\lambda)$ em $S_{\rho, \delta}^{0}$.

\section{Proposição 4.7. As funçôes}

1. $\lambda \in \Lambda \mapsto\langle\lambda\rangle b^{N}(\lambda) \in S_{\rho, \delta}^{0}$

2. $\lambda \in \Lambda \mapsto\langle\lambda\rangle r^{N}(\lambda) \in S_{\rho, \delta}^{m-N(\rho-\delta)}$

são contínuas e limitadas.

Demonstração. Escrevendo

$$
\langle\lambda\rangle b^{N}(\lambda)=\sum_{j<N}\langle\lambda\rangle b_{j}(\lambda)
$$

verificamos o primeiro item levando em conta a Proposição 4.6 e a continuidade das inclusões $S_{\rho, \delta}^{-j(\rho-\delta)} \hookrightarrow S_{\rho, \delta}^{0}$.

Para o segundo item, considere

$$
q_{N}(\lambda)=\sum_{|\alpha|<N} \frac{1}{\alpha !} \partial_{\xi}^{\alpha}(a-\lambda) D_{x}^{\alpha} b^{N}(\lambda)
$$

e escreva

$$
\langle\lambda\rangle r^{N}(\lambda)=\left[\langle\lambda\rangle\left((a-\lambda) \# b^{N}(\lambda)-q_{N}(\lambda)\right)\right]+\left[\langle\lambda\rangle\left(q_{N}(\lambda)-1\right)\right] .
$$

Assim bastará mostrar que cada termo entre colchetes é uma função contínua e limitada de $\Lambda$ em

$S_{\rho, \delta}^{m-N(\rho-\delta)}$ Defina

$$
T_{j, \alpha}(\lambda)=\frac{1}{\alpha !} \partial_{\xi}^{\alpha}(a-\lambda) D_{x}^{\alpha} b_{j}(\lambda)
$$

desse modo

$$
q_{N}(\lambda)=\sum_{|\alpha|<N} \sum_{j<N} T_{j, \alpha}(\lambda)
$$

Notamos que a função $f^{\alpha}: S_{\rho, \delta}^{u} \times S_{\rho, \delta}^{v} \rightarrow S_{\rho, \delta}^{u+v-|\alpha|(\rho-\delta)}$ dada por $f^{\alpha}(p, q)=\frac{1}{\alpha !} \partial_{\xi}^{\alpha} p D_{x}^{\alpha} q$ é contínua para todo $u, v \in \mathbb{R}$. Assim, pela continuidade das aplicações

- $\lambda \in \Lambda \mapsto a-\lambda \in S_{\rho, \delta}^{m}$

- $\lambda \in \Lambda \mapsto\langle\lambda\rangle b_{j}(\lambda) \in S_{\rho, \delta}^{-j(\rho-\delta)}$

segue que

$$
\lambda \in \Lambda \mapsto\langle\lambda\rangle T_{j, \alpha}(\lambda)=f^{\alpha}\left(a-\lambda,\langle\lambda\rangle b_{j}(\lambda)\right) \in S_{\rho, \delta}^{m-(j+|\alpha|)(\rho-\delta)}
$$


é contínua. Observando que $j+|\alpha| \geq N$ implica $S_{\rho, \delta}^{m-(j+|\alpha|)(\rho-\delta)} \hookrightarrow S_{\rho, \delta}^{m-N(\rho-\delta)}$, fazemos a separação dos termos de ordem alta e baixa $(j+|\alpha|<N$ e $j+|\alpha| \geq N$, respectivamente)

$$
q_{N}(\lambda)=\sum_{j+|\alpha|<N} T_{j, \alpha}(\lambda)+\sum_{j+|\alpha| \geq N} T_{j, \alpha}(\lambda)
$$

Para segunda soma $j<N$ e $j+|\alpha| \geq N$ implicam $\alpha \neq 0$ e, portanto, $\partial^{\alpha}(a-\lambda)=\partial^{\alpha} a$. Destas observações, concluímos $\lambda \in \Lambda \mapsto\langle\lambda\rangle T_{j, \alpha}(\lambda) \in S_{\rho, \delta}^{m-N(\rho-\delta)}$ é contínuo e

$$
\left\{\langle\lambda\rangle T_{j, \alpha}(\lambda)=\frac{1}{\alpha !} \partial_{\xi}^{\alpha}(a-\lambda) D_{x}^{\alpha}\left(\langle\lambda\rangle b_{j}(\lambda)\right): \lambda \in \Lambda\right\}=f^{\alpha}\left(\{a\} \times B_{j}\right)
$$

onde $\left.B_{j}=\left\{\langle\lambda\rangle b_{j}(\lambda)\right): \lambda \in \Lambda\right\}$. Pela Proposição 4.6, o conjunto $B_{j}$ é limitado em $S_{\rho, \delta}^{-j(\rho-\delta)}$, logo $\{a\} \times B_{j}$ é limitado em $S_{\rho, \delta}^{m} \times S_{\rho, \delta}^{-j(\rho-\delta)}$. Como $f^{\alpha}$ é contínua temos que $f^{\alpha}\left(\{a\} \times B_{j}\right)$ é limitado em $S_{\rho, \delta}^{m-N(\rho-\delta)}$.

Analisaremos agora os termos de ordem alta $(j+|\alpha|<N)$. Reordenado a somatória, temos

$$
H_{N}=\sum_{j+|\alpha|<N} T_{j, \alpha}=\sum_{l=0}^{N-1} \sum_{j+|\alpha|=l} T_{j, \alpha}
$$

Como para $l=0$ temos $j=0$ e $\alpha=0$ podemos escrever

$$
H_{N}=T_{0,0}+\sum_{l=1}^{N-1} \sum_{j+|\alpha|=l} T_{j, \alpha}
$$

Separando os pares $(j, \alpha)$ onde $\alpha=0$ ficamos com

$$
H_{N}=T_{0,0}+\sum_{l=1}^{N-1}\left(T_{l, 0}+\sum_{\substack{|\alpha|+j=l, 0 \leq j<l}} T_{j, \alpha}\right)=T_{0,0}=1
$$

já que pela recursão na definição 4.3

$$
T_{l, 0}+\sum_{\substack{|\alpha|+j=l, 0 \leq j<l}} T_{j, \alpha}=0
$$

para todo $l \geq 1$.

Perceba que o termo $(a-\lambda) \# b^{N}(\lambda)-q_{N}(\lambda)$ é o resto da expansão assintótica da composição dos símbolos $a-\lambda$ e $b^{N}(\lambda)$. Portanto suas seminormas em $S_{\rho, \delta}^{m-N(\rho-\delta)}$ podem ser estimadas por seminormas de $\partial_{\xi}^{\gamma} a$ em $S_{\rho, \delta}^{m-N \rho}$ e de $\langle\lambda\rangle \partial_{x}^{\gamma} b^{N}$ em $S_{\rho, \delta}^{N \delta} \operatorname{com}|\gamma|=N$. Disto segue que este termo é limitado e contínuo.

De modo análogo mostra-se o conteúdo da Proposição 4.7 para os símbolos

- $\tilde{b}_{j+1}(\lambda)=-\sum_{\substack{|\alpha|+k=j+1 \\ 0 \leq k \leq j}}, \frac{1}{\alpha !} \partial_{\xi}^{\alpha} \tilde{b}_{k}(\lambda) D_{x}^{\alpha} a b_{0}(\lambda)$

- $\tilde{b}^{N}(\lambda)=\sum_{j<N} \tilde{b}_{j}(\lambda)$

- $\tilde{r}^{N}(\lambda)=\tilde{b}^{N}(\lambda) \#(a-\lambda)-1$

Teorema 4.8. Para cada $N$ satisfazendo $m-N(\rho-\delta)$ existe $R \geq 0$ tal que $(a-\lambda)$ é invertivel no produto \# para todo $\lambda \in \Lambda_{R}=\{z \in \Lambda:|z| \geq R\}$. Definindo $s^{N}(\lambda)=(a-\lambda)^{-\#}-b^{N}(\lambda)$, as funções 
- $\lambda \in \Lambda_{R} \mapsto\langle\lambda\rangle(a-\lambda)^{-\#} \in S_{\rho, \delta}^{0}$

- $\lambda \in \Lambda_{R} \mapsto\langle\lambda\rangle^{2} s^{N}(\lambda) \in S_{\rho, \delta}^{0}$

são contínuas e limitadas.

Demonstração. Pelo Teorema 3.17 no Capítulo 3, o conjunto $G=\left\{a \in S_{\rho, \delta}^{0}: \exists b \in S_{\rho, \delta}^{0}, a \# b=\right.$ $b \# a=1\}$ é aberto em $S_{\rho, \delta}^{0}$. Como $1 \in G$ existe $l \geq 0$ inteiro e $\varepsilon>0$ tais que

$$
1+\left\{a \in S_{\rho, \delta}^{0}: p_{l}^{0}(a)<\varepsilon\right\} \subset G
$$

Portanto, $1+a \in G$, para todo $a \operatorname{com} p_{l}^{0}(a)<\varepsilon$. Pela Proposição 4.7, temos $c_{l}=\sup \left\{p_{l}^{0}\left(\langle\lambda\rangle r^{N}(\lambda)\right)\right.$ : $\lambda \in \Lambda\}<+\infty$. Podemos, então, tomar $R_{1}$ tal que $\langle\lambda\rangle^{-1}<\frac{\varepsilon}{c_{l}+1}$ para todo $|\lambda| \geq R_{1}$. Logo

$$
p_{l}^{0}\left(r^{N}(\lambda)\right)<\varepsilon
$$

para todo $\lambda \in \Lambda$ com $|\lambda| \geq R_{1}$.

Assim, $1+r^{N}(\lambda) \in G$, para todo $\lambda \in \Lambda_{R_{1}}$. Da definição de $r^{N}$ segue

$$
(a-\lambda) \#\left[b^{N}(\lambda) \#\left(1+r^{N}(\lambda)\right)^{-\#}\right]=1
$$

e obtemos uma inversa à direita para $a-\lambda$. De modo análogo verificamos que existe $R_{2}$ tal que $\left(1+\tilde{r}^{N}(\lambda)\right)^{-\#} \# \tilde{b}^{N}(\lambda)$ é uma inversa à esquerda para $\lambda \in \Lambda_{R_{2}}$. Portanto $a-\lambda$ é invertível e

$$
(a-\lambda)^{-\#}=b^{N}(\lambda) \#\left(1+r^{N}(\lambda)\right)^{-\#} \in S_{\rho, \delta}^{0}
$$

sempre que $\lambda \in \Lambda_{R}$, onde $R=\max \left\{R_{1}, R_{2}\right\}$.

Como a inversão é contínua em $S_{\rho, \delta}^{0}$, pelos resultados da Seção 3.4, segue que $\lambda \in \Lambda_{R} \mapsto$ $\left(1+r^{N}(\lambda)\right)^{-\#} \in S_{\rho, \delta}^{0}$ é contínua e limitada. Logo

$$
\langle\lambda\rangle(a-\lambda)^{-\#}=\left(\langle\lambda\rangle b^{N}(\lambda)\right) \#\left(1+r^{N}(\lambda)\right)^{-\#}
$$

fica limitada e contínua na váriavel $\lambda \in \Lambda_{R}$. Usando a identidade

$$
\left(1+r^{N}(\lambda)\right)^{-\#}=1-r^{N}(\lambda) \#\left(1+r^{N}(\lambda)\right)^{-\#}
$$

temos

$$
(a-\lambda)^{-\#}-b^{N}(\lambda)=-b^{N}(\lambda) \# r^{N}(\lambda) \#\left(1+r^{N}(\lambda)\right)^{-\#}
$$

donde concluímos $\lambda \in \Lambda_{R} \mapsto\langle\lambda\rangle^{2} s^{N}(\lambda) \in S_{\rho, \delta}^{0}$ é limitada e contínua.

Vamos agora aproveitar o Teorema 4.8 para obter uma estimativa como a do Teorema 2.23 no Capítulo 2 (condição para um operador ter cálculo limitado) só que no contexto de símbolos ao invés de operadores. Iniciamos notando que a integral

$$
b_{f}^{N}(x, \xi)=\frac{1}{2 \pi i} \int_{\partial \Lambda} f(\lambda) b^{N}(x, \xi ; \lambda) d \lambda
$$

é absolutamente convergente quaisquer que sejam $x, \xi \in \mathbb{R}^{n}$ e $f \in H_{1}^{\infty}$ e define uma função de classe $C^{\infty}$. Isto é consequência de que as seminormas de $b^{N}(\lambda)$ decaem como $\langle\lambda\rangle^{-1}$.

Usando que as singularidades de $\lambda \mapsto b^{N}(\lambda)$ estão em $\Omega_{x, \xi}$ e a continuidade de $b^{N}$, temos o seguinte:

Proposição 4.9. Para cada $(x, \xi) \in \mathbb{R}^{n} \times \mathbb{R}^{n}$, existe uma vizinhança $V \subset \mathbb{R}^{n} \times \mathbb{R}^{n}$ de $(x, \xi)$ tal que

$$
b_{f}^{N}(y, \eta)=\frac{1}{2 \pi i} \int_{\partial \Omega_{x, \xi}} f(\lambda) b^{N}(y, \eta ; \lambda) d \lambda
$$


para todo $(y, \eta) \in V$.

Proposição 4.10. A função $b_{f}^{N}$ é um símbolo em $S_{\rho, \delta}^{0}$ e para cada seminorma $q$ de $S_{\rho, \delta}^{0}$ existe $c_{q}>0$ tal que

$$
q\left(b_{f}^{N}\right) \leq c_{q}\|f\|_{\infty}
$$

para toda $f \in H_{1}^{\infty}$.

Demonstração. Sejam $x, \xi \in \mathbb{R}^{n}$. Pela proposição 4.9 , temos que

$$
\partial_{\xi}^{\alpha} \partial_{x}^{\beta} b_{f}^{N}(y, \eta)=\frac{1}{2 \pi i} \int_{\partial \Omega_{x, \xi}} f(\lambda) \partial_{\xi}^{\alpha} \partial_{x}^{\beta} b^{N}(y, \eta ; \lambda) d \lambda
$$

quando $(y, \eta) \in V$. Fazendo $(y, \eta)=(x, \xi)$, obtemos a estimativa

$$
\left|\partial_{\xi}^{\alpha} \partial_{x}^{\beta} b_{f}^{N}(x, \xi)\right| \leq(2 \pi)^{-1}\left|\partial \Omega_{x, \xi}\right|\|f\|_{\infty} \sup _{\lambda \in \partial \Omega_{x, \xi}}\left|\partial_{\xi}^{\alpha} \partial_{x}^{\beta} b^{N}(x, \xi ; \lambda)\right| .
$$

Notando que $\partial \Omega_{x, \xi}$ é formada por um arco de circunferência e dois segmentos de reta podemos calcular seu comprimento

$$
\left|\partial \Omega_{x, \xi}\right|=2 \theta(2|a(x, \xi)|)+2|a(x, \xi)|+2|a(x, \xi)|=4(1+\theta)|a(x, \xi)| .
$$

Por outro lado segue da proposição 4.5

$$
\left|\partial_{\xi}^{\alpha} \partial_{x}^{\beta} b^{N}(x, \xi ; \lambda)\right| \leq M\left|(a(x, \xi)-\lambda)^{-1}\right|\langle\xi\rangle^{-\rho|\alpha|+\delta|\beta|}
$$

enquanto a estimativa do primeiro item da proposição 4.2 (para $\alpha=\beta=0$ ) implica $\mid(a(x, \xi)-$ $\lambda)\left.^{-1}\left|\leq c_{0,0}\right| a(x, \xi)\right|^{-1}$. Portanto

$$
\left|\partial_{\xi}^{\alpha} \partial_{x}^{\beta} b^{N}(x, \xi ; \lambda)\right| \leq M_{1}|a(x, \xi)|^{-1}\langle\xi\rangle^{-\rho|\alpha|+\delta|\beta|} .
$$

Retornado desigualdade (4.2) concluímos

$$
\begin{aligned}
\left|\partial_{\xi}^{\alpha} \partial_{x}^{\beta} b_{f}^{N}(x, \xi)\right| & \leq 4(1+\theta) M_{1}(2 \pi)^{-1}\|f\|_{\infty}|a(x, \xi)||a(x, \xi)|^{-1}\langle\xi\rangle^{-\rho|\alpha|+\delta|\beta|} \\
& =c_{q}\|f\|_{\infty}\langle\xi\rangle^{-\rho|\alpha|+\delta|\beta|} .
\end{aligned}
$$

Teorema 4.11. Seja a um simbolo $H S$. Existe $c \in \mathbb{R}$ tal que, definindo $a_{c}=a+c e$

$$
f\left(a_{c}\right)=\frac{1}{2 \pi i} \int_{\partial \Lambda} f(\lambda)\left(a_{c}-\lambda\right)^{-\#} d \lambda,
$$

temos $f\left(a_{c}\right) \in S_{\rho, \delta}^{0}$ e $q\left(f\left(a_{c}\right)\right) \leq d_{q}\|f\|_{\infty}$, para toda $f \in H_{1}^{\infty}$ e seminorma $q$ de $S_{\rho, \delta}^{0}$.

Demonstração. Seja $R$ como no Teorema 4.8 e escolha $c \geq R$. Deste modo $a+c-\lambda=a-(\lambda-c)$ é inversível sempre que $\lambda-c \in \Lambda_{R}$ e, além disso

$$
\left(a_{c}-\lambda\right)^{-\#}=b^{N}(\lambda-c)+s^{N}(\lambda-c) .
$$

Note que como $c \geq R$ temos $\Lambda \subset \Lambda_{R}+c$ e por isso a identidade acima vale para todo $\lambda \in \Lambda$. Do Teorema 4.8 também segue que cada símbolo em (4.3) multiplicado por $f \in H_{1}^{\infty}$ é integrável e, assim

$$
\int_{\partial \Lambda} f(\lambda)\left(a_{c}-\lambda\right)^{-\#} d \lambda=\int_{\partial \Lambda} f(\lambda) b^{N}(\lambda-c) d \lambda+\int_{\partial \Lambda} f(\lambda) s^{N}(\lambda-c) d \lambda .
$$

A segunda integral do lado direito é estimada facilmente já que as seminormas de $s^{N}$ decaem como $\langle\lambda\rangle^{-2}$. Para outra notamos que $f(.) b^{N}(.-c)$ é analítica entre os contornos $\partial \Lambda$ e $\partial \Lambda+c$. Logo pelo 
Teorema de Cauchy temos

$$
\int_{\partial \Lambda} f(\lambda) b^{N}(\lambda-c) d \lambda=\int_{\partial \Lambda+c} f(\lambda) b^{N}(\lambda-c) d \lambda .
$$

Mas a identidade $\int_{\gamma} f(z) d z=\int_{\gamma-c} f(z+c) d z$ implica

$$
\int_{\partial \Lambda} f(\lambda) b^{N}(\lambda-c) d \lambda=\int_{\partial \Lambda} f(\lambda+c) b^{N}(\lambda) d \lambda=b_{g}^{N}
$$

com $g(\lambda)=f(\lambda+c)$. Aplicando a Proposição 4.10 a $g$ e observando que $\|g\|_{\infty} \leq\|f\|_{\infty}$, obtemos

$$
q\left(b_{g}^{N}\right) \leq c_{q}\|f\|_{\infty}
$$

finalizando a demonstração.

\subsection{Cálculo para o operador}

No restante fixamos $0 \leq \delta<\rho \leq 1$ e assumimos que $E$ é um espaço de Banach tal que

1. $E \hookrightarrow \mathcal{S}^{\prime}$ é contínua e $\mathcal{S}$ é um subconjunto denso de $E$;

2. op : $S_{\rho, \delta}^{0} \rightarrow \mathcal{B}(E)$ é contínua;

Denotamos $o_{E}[b] \in \mathcal{B}(E)$ para $b \in S_{\rho, \delta}^{0}$.

Nestas condições temos o teorema principal da dissertação:

Teorema 4.12. Suponha que a é um símbolo HS. O fecho A do operador pseudodiferencial op $[a]$ : $\mathcal{S} \subset E \rightarrow E$ existe $e$, para algum $c \in \mathbb{R}, A+c$ é setorial com cálculo $H^{\infty}$.

A seguir op $[a]$ representa o operador agindo tanto em $\mathcal{S}$ como em $\mathcal{S}^{\prime}$.

Definição 4.13. O operador maximal $A_{1}$ de op $[a]: \mathcal{S} \rightarrow \mathcal{S}$ em $E$ é definido por

$$
D\left(A_{1}\right)=\{u \in E: o p[a] u \in E\}
$$

e $A_{1} u=o p[a] u$.

Por definição, $A_{1}$ é uma extensão de $o p[a]: \mathcal{S} \subset E \rightarrow E$.

Proposição 4.14. O operador maximal $A_{1}: D\left(A_{1}\right) \subset E \rightarrow E$ é fechado.

Demonstração. Sejam $u_{n} \in D\left(A_{1}\right), u, v \in E$ tais que $u_{n} \stackrel{E}{\longrightarrow} u$ e $A_{1} u_{n} \stackrel{E}{\longrightarrow} v$. Como $E \hookrightarrow \mathcal{S}^{\prime}$ é contínua temos $u_{n} \stackrel{\mathcal{S}^{\prime}}{\longrightarrow} u$ e $A_{1} u_{n} \stackrel{\mathcal{S}^{\prime}}{\longrightarrow} v$. Por outro lado, a continuidade de op $[a]: \mathcal{S}^{\prime} \rightarrow \mathcal{S}^{\prime}$ implica $A_{1} u_{n}=o p[a] u_{n} \stackrel{\mathcal{S}^{\prime}}{\longrightarrow} o p[a] u$. Logo op $[a] u=v \in E$ o que mostra $u \in D\left(A_{1}\right)$ e $A_{1} u=v$.

Lembramos que o gráfico de $A=\overline{o p[a]}$ é igual ao fecho do gráfico de $o p[a]$ em $E \times E$. Dito de outra forma, $u \in D(A)$ se, e somente se, existir $u_{n} \in \mathcal{S}$ e $v \in E$ tais que $u_{n} \stackrel{E}{\longrightarrow} u$ e op $[a] u_{n} \stackrel{E}{\longrightarrow} v$. Neste caso $A u=v$.

Proposição 4.15. Seja $\lambda \in \mathbb{C}$. Se existe $(a-\lambda)^{-\#} \in S_{\rho, \delta}^{0}$ então $\lambda \in \rho(A)$ e

$$
o p_{E}\left[(a-\lambda)^{-\#}\right]=(A-\lambda)^{-1}
$$

Demonstração. Basta mostrar que $B=o p_{E}\left[(a-\lambda)^{-\#}\right]$ satisfaz $B(A-\lambda) \subset I$ e $(A-\lambda) B=I$. 
1. Para $u \in D(A), B(A-\lambda) u=u$

Dado $u \in D(A)$ tome $u_{n} \in \mathcal{S}$ tal que $u_{n} \stackrel{E}{\longrightarrow} u$ e $A u_{n} \stackrel{E}{\longrightarrow} A u$. Assim, $(A-\lambda) u_{n} \stackrel{E}{\longrightarrow}(A-\lambda) u$ e como $B \in \mathcal{B}(E)$ segue $B(A-\lambda) u_{n} \stackrel{E}{\longrightarrow} B(A-\lambda) u$. Para $u_{n} \in \mathcal{S}$ temos

$$
B(A-\lambda) u_{n}=o p\left[(a-\lambda)^{-\#}\right] o p[a-\lambda] u_{n}=u_{n} .
$$

Logo $B(A-\lambda) u_{n} \stackrel{E}{\longrightarrow} u$. Portanto $u=B(A-\lambda) u$.

2. Para $u \in E,(A-\lambda) B u=u$

Dado $u \in E$, a densidade de $\mathcal{S}$ em $E$ fornece $u_{n} \in \mathcal{S}$ com $u_{n} \stackrel{E}{\longrightarrow} u$. Como $B \in \mathcal{B}(E)$, $B u_{n} \stackrel{E}{\longrightarrow} B u$. Novamente de $u_{n} \in \mathcal{S}$ temos

$$
(A-\lambda) B u_{n}=u_{n}
$$

dando que $(A-\lambda) B u_{n} \stackrel{E}{\longrightarrow} u$. Como $A-\lambda$ é fechado temos $B u \in D(A)$ e $(A-\lambda) B u=u$.

Proposição 4.16. Existe $c \in \mathbb{R}$ para o qual $A+c$ é setorial.

Demonstração. Seja $R$ como no Teorema 4.8 e escolha $c \geq R$. Assim $\left(a_{c}-\lambda\right)^{-\#} \in S_{\rho, \delta}^{0}$ para todo $\lambda \in \Lambda$ e a Proposição 4.15 implica $(A+c-\lambda)^{-1}=o p_{E}\left[\left(a_{c}-\lambda\right)^{-\#}\right]$. Por conta da continuidade de op : $S_{\rho, \delta}^{0} \rightarrow \mathcal{B}(E)$, existe uma seminorma $q$ tal que

$$
\left\|o p_{E}\left[\left(a_{c}-\lambda\right)^{-\#}\right]\right\| \leq M q\left(\left(a_{c}-\lambda\right)^{-\#}\right)
$$

Do Teorema 4.8 também segue a estimativa

$$
q\left(\left(a_{c}-\lambda\right)^{-\#}\right) \leq L_{q}\langle\lambda-c\rangle^{-1} \leq L_{q}^{\prime}\langle\lambda\rangle^{-1}
$$

Logo,

$$
\left\|(A+c-\lambda)^{-1}\right\| \leq M^{\prime}\langle\lambda\rangle^{-1}
$$

para todo $\lambda \in \Lambda$ e, portanto, $A+c$ é setorial de ângulo $\theta$ (lembre que $\Lambda=\mathbb{C} \backslash \Sigma_{\theta}$ ).

Pondo $A_{c}=A+c$ ficamos com um cálculo para $A_{c}$ dado pela integral de Cauchy

$$
f\left(A_{c}\right)=\frac{1}{2 \pi i} \int_{\partial \Lambda} f(\lambda)\left(A_{c}-\lambda\right)^{-1} d \lambda
$$

Proposição 4.17. Se $f \in H_{1}^{\infty}$,

$$
f\left(A_{c}\right)=o p_{E}\left[f\left(a_{c}\right)\right]
$$

Demonstração. A maneira mais rápida de mostrar esta proposição é usar um teorema sobre integração em espaços de Fréchet que permite passar transformações lineares contínuas para dentro da integral. Nesse caso a integrabilidade de $f\left(a_{c}\right)$ em $S_{\rho, \delta}^{0}$ e a continuidade de $o p: S_{\rho, \delta}^{0} \rightarrow \mathcal{B}(E)$ implicam a igualdade do enunciado. O leitor interessado pode consultar [Rud91].

Finalmente demonstramos o teorema principal.

Demonstração do Teorema 4.12. Segue do Teorema 4.11 e da Proposição 4.17 que

$$
\left\|f\left(A_{c}\right)\right\|=\left\|o p_{E}\left[f\left(a_{c}\right)\right]\right\| \leq c q\left(f\left(a_{c}\right)\right) \leq c d_{q}\|f\|_{\infty}
$$

De acordo com o Teorema 2.23 isto é suficiente para mostrar que $A_{c}$ tem cálculo $H^{\infty}$. 


\section{Capítulo 5}

\section{Aplicações}

Neste capítulo apresentamos uma aplicação do cálculo ao problema de regularidade maximal. Na Seção 5.1 damos a formulação completa do problema de regularidade maximal para um operador linear geral agindo num espaço de Banach. Vale a pena mencionar que estabelecer a regularidade maximal de um operador linear tem consequências na existência e regularidade de soluções de equações parabólicas não lineares. Na Seção 5.2 definimos o semigrupo gerado por um operador setorial com ângulo estritamente menor do que $\pi / 2$ usando apenas o cálculo funcional básico $\left(H_{1}^{\infty}\right)$ desenvolvido no Capítulo 2. Com a existência do cálculo funcional $H^{\infty}$, deduzimos a hipótese sobre potências imaginárias do Teorema de Dore e Venni para chegar na regularidade maximal do operador. Ao final desta seção introduzimos os espaços UMD para os quais os resultados se aplicam. Na última seção apresentamos uma coleção de exemplos de operadores com símbolos HS e indicamos algumas consequências das discussões anteriores.

\subsection{Regularidade Maximal}

Considere o problema de valor inicial

$$
\begin{aligned}
\partial_{t} v(t, x)+\Delta v(t, x) & =g(t, x) \quad 0<t<T, \quad x \in \mathbb{R}^{n} \\
v(0, x) & =0 \quad x \in \mathbb{R}^{n}
\end{aligned}
$$

Definindo as funções $u, f:[0, T] \rightarrow X$ por $u(t)=v(t, \cdot)$ e $f(t)=g(t, \cdot)$, onde $X$ é um espaço de funções em $\mathbb{R}^{n}$ apropriado, podemos reescrever o problema como uma equação diferencial ordinária com condição inicial nula

$$
\begin{aligned}
u^{\prime}(t)+A u(t) & =f(t) \quad 0<t<T \\
u(0) & =0
\end{aligned}
$$

designaremos este problema como PCA ou Problema de Cauchy Abstrato.

Para este problema reformulado iremos discutir o conceito de regularidade maximal $L^{p}$.

Fixamos $1<p<\infty$, um intervalo compacto $I=[0, T]$ e $X$ um espaço de Banach. Para definir o conceito de regularidade maximal $L^{p}$, usaremos as funções $L^{p}(I ; X)$ e $W_{p}^{1}(I ; X)$ definidas na Seção 2.2 do Capítulo 2.

Definição 5.1. Seja $A: D(A) \subset X \rightarrow X$ um operador linear. Dizemos que A possui regularidade maximal se estão satisfeitas as seguintes condiçôes:

- Para cada $f \in L^{p}(I ; X)$ existe uma única função $u_{f}: I \rightarrow X$ tal que

$-u_{f} \in W_{p}^{1}(I ; X)$;

- $u_{f}(t) \in D(A)$, para quase todo $t \in I$;

- $u_{f}^{\prime}+A u_{f}(t)=f(t)$, para quase todo $t \in I$; 
$-u_{f}(0)=0 ;$

Chamamos tal uf de solução $L^{p}$ do PCA.

- As aplicações $f \mapsto u_{f}, f \mapsto u_{f}^{\prime}$ e $f \mapsto A u_{f}$ são contínuas de $L^{p}(I ; X)$ em si mesmo.

Assim, $A$ possui regularidade maximal se o problema é bem posto em $L^{p}$. Um outro modo de visualizar o problema é por meio das aplicações definidas a seguir. Sejam $B: D(B) \subset L^{p} \rightarrow L^{p}$ e $\mathcal{A}: D(\mathcal{A}) \subset L^{p} \rightarrow L^{p}$ os operadores lineares com domínios

$$
D(B)=\left\{u \in W_{p}^{1}: u(0)=0\right\}
$$

e

$$
D(\mathcal{A})=\left\{u \in L^{p}: u(t) \in D(A) q . s ., \quad t \mapsto A u(t) \in L^{p}\right\}
$$

dados por $B u=u^{\prime}$ e $(\mathcal{A} u)(t)=A(u(t))$. Então o operador $A$ possui regularidade maximal se, e somente se, $B+\mathcal{A}: D(B) \cap D(\mathcal{A}) \subset L^{p} \rightarrow L^{p}$ tem uma inversa contínua. Note que nesta formulação parece que somos mais exigentes supondo que $t \mapsto A u(t)$ pertence a $L^{p}$. Porém, se $u$ é uma solução $L^{p}$ temos que $A u=f-u^{\prime} \in L^{p}$.

\subsection{Aplicações}

Podemos mostrar que operadores que possuem regularidade maximal são geradores de semigrupo analítico. Vamos definir abaixo essa importante classe de operadores.

Seja $A$ um operador setorial de ângulo $\omega<\pi / 2$. Se $\sigma<\pi / 2$ então as funções $f_{\lambda}(z)=e^{-\lambda z}$ pertencem a $H_{1}^{\infty}\left(\Sigma_{\sigma}\right)$ sempre que $\lambda \in \Sigma_{\pi / 2-\sigma}$. Definindo $e^{-\lambda A}=f_{\lambda}(A)$ segue da propriedade multiplicativa do cálculo

$$
e^{-\lambda A} e^{-\mu A}=f_{\lambda}(A) f_{\mu}(A)=f_{\lambda} f_{\mu}(A)=e^{-(\lambda+\mu) A}
$$

mostrando que os operadores $e^{-\lambda A}$ assim definidos formam um semigrupo. Podemos então definir gerador de semigrupo analítico da seguinte forma:

Definição 5.2. Dizemos que $A: D(A) \subset X \rightarrow X$ é um gerador de semigrupo analitico se existe $c \in \mathbb{R}$ tal que $c-A$ é setorial de ângulo $\omega<\pi / 2$. Neste caso $e^{t A} \doteq e^{t(A-c)} e^{c t}$.

Se $-A$ é gerador de um semigrupo analítico então o PCA tem formalmente uma solução definida por

$$
u(t)=\int_{0}^{t} e^{-(t-s) A} f(s) d s
$$

Podemos mostrar que o problema de regularidade maximal se reduz a mostrar que a solução acima pertence a $W_{p}^{1}(I ; X)$.

Uma abordagem para resolver este problema é através das potências imaginárias $A^{i t}$ que também podem ser definidas usando o cálculo funcional. Em geral se $A$ é um operador setorial de ângulo $0<\omega<\pi$ é possível definir $A^{i t}, t \in \mathbb{R}$, como operadores fechados não necessariamente limitados. É interessante observar que definindo $f_{t}: \Sigma_{\sigma} \rightarrow \mathbb{C}(\omega<\sigma)$, por $f_{t}(z)=z^{i t}$, o operador usado na demonstração do Teorema 2.23 do Capítulo 2

$$
\bar{\Phi}_{A}\left(f_{t}\right)=\Phi_{A}\left(f_{t}(1+z)^{-1}\right)(1+A) x, \quad x \in D(A)
$$

permite definir as potências imaginárias visto que $\left\|f_{t}\right\|_{\infty} \leq e^{|t| \sigma}$. Quando $A$ possui cálculo $H^{\infty}\left(\Sigma_{\sigma}\right)$ é imediato que os operadores $A^{i t}=f_{t}(A)$ são limitados e satisfazem

$$
\left\|A^{i t}\right\| \leq C_{\sigma} e^{|t| \sigma}
$$

Supondo ainda $\sigma<\pi / 2$ e que $X$ é UMD (explicado mais adiante), o conhecido Teorema de Dore e Venni enunciado a seguir implica regularidade maximal. 
Teorema 5.3 (Dore-Venni). Sejam $X$ um espaço UMD e $A: D(A) \subset X \rightarrow X$ um operador setorial de ângulo $0<\omega<\pi / 2$. Suponha que existam $C>0$ e $\omega<\sigma<\pi / 2$ tais que

$$
\left\|A^{i t}\right\| \leq C e^{|t| \sigma}
$$

para todo $t \in \mathbb{R}$. Então A possui regularidade maximal.

A demonstração deste Teorema é longa mas sua ideia fundamental consiste em provar

$$
(B+\mathcal{A})^{-1}=\frac{1}{2 i} \int_{a-i \infty}^{a+i \infty} \frac{B^{-z} \mathcal{A}^{z-1}}{\sin (\pi z)} d z
$$

onde $a \in] 0,1[$.

Damos a seguir uma breve explicação dos espaços UMD. Seja $X$ um espaço de Banach. Formalmente a transformada de Fourier de funções vetoriais em $L^{1}(\mathbb{R} ; X)$ é definida como no caso escalar. Dada $m: \mathbb{R} \rightarrow \mathbb{C}$ mensurável e limitada definimos o operador $m(D): C_{c}^{\infty}(\mathbb{R} ; X) \rightarrow C(\mathbb{R} ; X)$ através da fórmula

$$
m(D)=\mathcal{F}^{-1}(m \mathcal{F})
$$

chamado de Multiplicador de Fourier (Fourier Multiplier). No caso escalar $X=\mathbb{C}$ o Teorema de Miklhin afirma que, sob certas condições na função $m$, o operador $m(D)$ é limitado em $L^{p}$. Este tipo de resultado é muito usado tanto na obtenção de estimativas para equações elípticas como no estudo de regularidade maximal de equações de evolução. Aqui estamos interessados apenas em dar uma caracterização dos espaços UMD.

Definição 5.4. Um espaço de Banach $X$ é UMD se para a função $m: \mathbb{R} \rightarrow \mathbb{R}$ dada por $m(\xi)=1$, se $\xi \geq 0$ e $m(\xi)=-1$, se $\xi<0$, o operador $m(D)$ é limitado em $L^{2}$, isto é,

- $m(D) C_{c}^{\infty}(\mathbb{R} ; X) \subset L^{2}$

- $\|m(D)\|_{L^{2}} \leq C\|u\|_{L^{2}}$

para todo $u \in C_{c}^{\infty}(\mathbb{R} ; X)$.

Como exemplos de espaços UMD temos $L^{p}\left(\mathbb{R}^{n}\right)$ e $W_{p}^{s}\left(\mathbb{R}^{n}\right)$ para $1<p<\infty$ e $s \in \mathbb{R}$. É conhecido que espaços UMD são reflexivos e, por isso, os espaços $L^{1}\left(\mathbb{R}^{n}\right), L^{\infty}\left(\mathbb{R}^{n}\right)$ não são UMD.

\subsection{Exemplos}

Nesta seção, apresentamos exemplos de operadores com a propriedade HS.

Exemplo 1. Seja $P=\sum_{|\alpha| \leq m} c_{\alpha}(x) \partial^{\alpha}$ um operador diferencial parcial linear em $\mathbb{R}^{n}$ com coeficientes suaves e derivadas de qualquer ordem limitadas. Neste caso a $(x, \xi)=\sum_{|\alpha| \leq m} i^{|\alpha|} c_{\alpha}(x) \xi^{\alpha}$ é um símbolo em $S_{1,0}^{m}$ do operador $P$, isto é, $P u=o p[a] u$, para todo $u \in \mathcal{S}$. Se, além disso, a é um símbolo HS com ângulo $\theta<\pi / 2$ concluímos que o fecho do operador $P: \mathcal{S} \subset L^{p}\left(\mathbb{R}^{n}\right) \rightarrow L^{p}\left(\mathbb{R}^{n}\right)$ tem regularidade maximal $L^{q}(1<p, q<\infty)$.

Exemplo 2. O simbolo $a(\xi)=1+|\xi|^{2}$ é $H S$ com qualquer ângulo $0<\theta<\pi$ e corresponde ao operador diferencial $1-\Delta$. No espaço $X=L^{p}\left(\mathbb{R}^{n}\right)$ o domínio de op $[a]$ é o espaço de Sobolev $W_{p}^{2}\left(\mathbb{R}^{n}\right)$. Escolhendo $\theta<\pi / 2$ obtemos a regularidade maximal do operador $-\Delta: W_{p}^{2}\left(\mathbb{R}^{n}\right) \rightarrow L^{p}\left(\mathbb{R}^{n}\right)$. Isto segue pois quando o intervalo $[0, T]$ é limitado basta mostrar a regularidade maximal de um dos operadores $A$ ou $A+c$ para obter o mesmo para o outro. 


\section{Referências Bibliográficas}

[ABHN11] Wolfgang Arendt, Charles J. K. Batty, Matthias Hieber e Frank Neubrander. Vectorvalued Laplace transforms and Cauchy problems, volume 96 of Monographs in Mathematics. Birkhäuser/Springer Basel AG, Basel, second edição, 2011. 4

[Bea77] Richard Beals. Characterization of pseudodifferential operators and applications. Duke Math. J., 44(1):45-57, 1977. 19

[BSS10] Olesya Bilyj, Elmar Schrohe e Jörg Seiler. $H_{\infty}$-calculus for hypoelliptic pseudodifferential operators. Proc. Amer. Math. Soc., 138(5):1645-1656, 2010. 1

[DS58] Nelson Dunford e Jacob T. Schwartz. Linear Operators. I. General Theory. With the assistance of W. G. Bade and R. G. Bartle. Pure and Applied Mathematics, Vol. 7. Interscience Publishers, Inc., New York; Interscience Publishers, Ltd., London, 1958. 1

[DV87] Giovanni Dore e Alberto Venni. On the closedness of the sum of two closed operators. Math. Z., 196(2):189-201, 1987. 1

[Fef73] Charles Fefferman. $L^{p}$ bounds for pseudo-differential operators. Israel J. Math., 14:413417, 1973. 16

[H6̈5] Lars Hörmander. Pseudo-differential operators. Comm. Pure Appl. Math., 18:501-517, 1965. 1

[H6̈7] Lars Hörmander. Pseudo-differential operators and hypoelliptic equations. páginas 138-183, 1967. 2

[Haa06] Markus Haase. The functional calculus for sectorial operators, volume 169 of Operator Theory: Advances and Applications. Birkhäuser Verlag, Basel, 2006. 2

[Kg81] Hitoshi Kumano-go. Pseudodifferential operators. MIT Press, Cambridge, Mass.London, 1981. Translated from the Japanese by the author, Rémi Vaillancourt and Michihiro Nagase. 13, 21

[KN65] J. J. Kohn e L. Nirenberg. An algebra of pseudo-differential operators. Comm. Pure Appl. Math., 18:269-305, 1965. 1

[McI86] Alan McIntosh. Operators which have an $H_{\infty}$ functional calculus. Em Miniconference on operator theory and partial differential equations (North Ryde, 1986), volume 14 of Proc. Centre Math. Anal. Austral. Nat. Univ., páginas 210-231. Austral. Nat. Univ., Canberra, 1986. 1

[PS16] Jan Prüss e Gieri Simonett. Moving interfaces and quasilinear parabolic evolution equations, volume 105. Springer, 2016. 2

[Rud91] Walter Rudin. Functional analysis. International Series in Pure and Applied Mathematics. McGraw-Hill, Inc., New York, second edição, 1991. 1, 29 
[Sch95] Elmar Schrohe. Fréchet algebras of pseudodiferential operators and boundary value problems, 1995. 19

[Tau05] Daniel Victor Tausk. Integration on banach spaces valued functions, 2005. 4

[Wae71] Lucien Waelbroeck. Topological vector spaces and algebras. Lecture Notes in Mathematics, Vol. 230. Springer-Verlag, Berlin-New York, 1971. 18

[Won14] M. W. Wong. An introduction to pseudo-differential operators, volume 6 of Series on Analysis, Applications and Computation. World Scientific Publishing Co. Pte. Ltd., Hackensack, NJ, third edição, 2014. 21

[Yag10] Atsushi Yagi. Abstract parabolic evolution equations and their applications. Springer Monographs in Mathematics. Springer-Verlag, Berlin, 2010. 1

[Yos95] Kosaku Yosida. Functional analysis. Classics in Mathematics. Springer-Verlag, Berlin, 1995. Reprint of the sixth (1980) edition. 4 